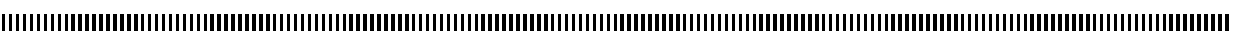

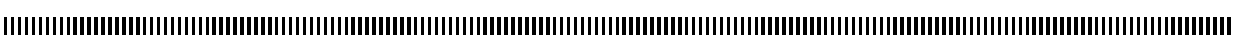
|

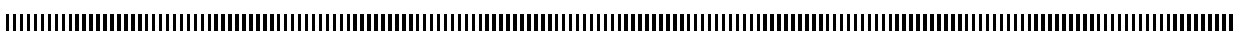

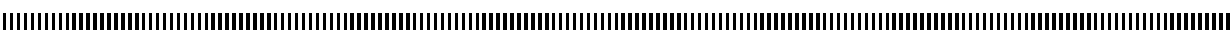

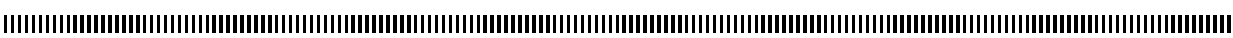

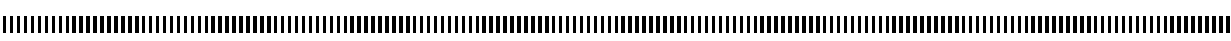

\title{
Nearly recombining processes and the calculation of expectations
}

\author{
Imme van den Berg a, ${ }^{*}$ Elsa Amaro b \\ a Departamento de Matemática, \\ Universidade de Évora, Colégio Luis António Verney, \\ 7000-671 Évora, PORTUGAL \\ * Corresponding author : ivdb@uevora.pt \\ b Escola Secundária de Reguengos de Monsaraz, \\ PORTUGAL \\ elsa.amaro@oninet.pt
}

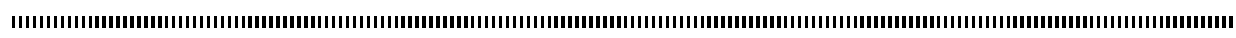

ABSTRACT. In the context of Nonstandard Analysis, we study stochastic difference equations with infinitesimal time-steps. In particular we give a necessary and sufficient condition for a solution to be nearly-equivalent to a recombining stochastic process. The characterization is based upon a partial differential equation involving the trend and the conditional variance of the original process. An analogy with Ito's Lemma is pointed out. As an application we obtain a method for approximation of expectations, in terms of two ordinary differential equations, also involving the trend and the conditional variance of the original process, and of Gaussian integrals.

RÉSUMÉ. Dans le contexte de l'Analyse Nonstandard, nous étudions des équations différentielles stochastiques avec des pas infiniment petits. En particulier, nous formulons une condition nécessaire et suffisante pourqu'une solution soit presque-équivalente à un processus stochastique recombinant. La caractérisation est donnée par une équation aux dérivées partielles de la tendance et de la variance conditionnelle du processus de départ. Nous indiquons une analogie avec le Lemme d'Ito. Nous appliquons cette caractérisation au problème de la détermination d'espérances pour le processus de départ. En fait, on obtient une approximation infinitésimale en resolvant deux équations différentielles ordinaires, également de la tendance et de la variance conditionnelle de ce processus, et en calculant une intégrale de Gauss.

KEYWORDS : Finite stochastic processes, recombination, near-equivalence, stroboscopy, expectations, Ito's Lemma

MOTS-CLÉS : Processus stochastiques finis, recombinant, presque-équivalence, espérances, Lemme d'Ito

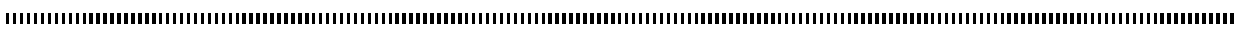




\section{Introduction}

We study in the context of Nonstandard Analysis a class of stochastic processes which are nearly-equivalent to recombining processes, otherwise said, processes which are nearlyequivalent to deterministic functions of time $t$ and the Wiener Walk $W^{1}$.

The Wiener Walk $W$ is given by the initial condition $W(0)=0$, and has independent increments $\delta W_{t}= \pm \sqrt{\delta t}$, where $\delta t$ is a fixed infinitesimal time-interval. The processes in question satisfy a stochastic difference equation of type

$$
\delta X_{t}=\mu\left(t, X_{t}\right) \delta t \pm \sigma\left(t, X_{t}\right) \sqrt{\delta t}
$$

here $\mu$ is the trend and $\sigma^{2}$ the conditional variance of the process $X$, and $t$ is of the form $t=k \delta t$, where $k$ is an integer and $t \leq T$, for some standard $T$. These discrete processes with infinitesimal increments are, in a sense, imitations of continuous-time processes, given by stochastic differential equations. In particular, under rather mild conditions on $\mu$ and $\sigma$, almost all of their trajectories are $S$-continuous - a nonstandard notion of nearcontinuity shared by standard continuous functions - , and also the laws of their stochastic variables may be $S$-continuous. Such processes have been rather intensively studied within Nonstandard Analysis, for instance in [2][12][18][17][1][27][22][20][4] and [7]. Well-known introductions to the classical approach are the books by Øksendal [23] and Protter [24].

A process is said to be recombining if, like in the case of the Wiener Walk, an upward movement followed by a downward movement yields the same result as a downward movement followed by an upward movement. This leads to a property of pathindependence: starting at a given initial point, every trajectory with the same number of upward movements and downward movements ends at the same point.

The notion of near-equivalence was introduced in [22]. In particular, if two processes $X$ and $Y$ are nearly-equivalent, the expectations $E F\left(X_{t}\right)$ and $E F\left(Y_{t}\right)$ of limited and $S$ continuous functions $F$ of their respective stochastic variables are infinitely close. Here lies a principal motivation of our work, for it induces an effective strategy to determine an infinitesimal approximation of expectations of stochastic variables $F\left(X_{t}\right)$ related to the stochastic difference equation (1), whenever its solution $X$ is nearly-equivalent to a recombining process $Y$. Indeed, for non-infinitesimal $t$ an infinitesimal approximation of the stochastic variables of the recombining process $Y$ may be determined by solving two ordinary differential equations, in terms of the trend $\mu$ and the conditional variance $\sigma^{2}$ of the process $X$, while their laws are binomial, hence almost Gaussian. Then $E F\left(X_{t}\right)$ will be infinitely close to a Riemann-sum, and in case of convergence, to an improper Riemann-integral (see formulae (6) and (7) and Theorem 6.4).

In this article we present a construction which associates a recombining process $Y$ to any process $X$ given by equation (1). The Main Theorem gives necessary and sufficient

1. An overheard occasional discussion remark by Claude Lobry happened to be the incentive for this study of recombining processes. Claudy Lobry also animated a workgroup on discrete stochastic processes leading to the expository text of Benoit [3]. This contains a characterization of nearlyequivalent processes which is essential for this article. 
conditions for the processes $X$ and $Y$ to be nearly-equivalent. Indeed, it is needed that $\mu$ and $\sigma$ satisfy the partial differential equation

$$
\sigma \frac{\partial \mu}{\partial X}-D \sigma=0
$$

where $D=\mu \frac{\partial}{\partial X}+\frac{\partial}{\partial t}+\frac{\sigma^{2}}{2} \frac{\partial^{2}}{\partial X^{2}}$ is the operator of Dynkin. The remaining conditions are regularity conditions, on existence, continuity and boundedness of some partial derivatives of $\mu$ and $\sigma$. Notice some similarity between (2) and the Cauchy-Riemann conditions for path-independence, where to the operator $\mu \frac{\partial}{\partial X}$ are added the terms $\frac{\partial}{\partial t}$ and $\frac{\sigma^{2}}{2} \frac{\partial^{2}}{\partial X^{2}}$.

The necessary part of the Main Theorem is in a sense an obvious consequence of the classical Lemma of Ito. Ito's Lemma, applied to stochastic differential equations $d X_{t}=\mu\left(t, B_{t}\right) d t+\sigma\left(t, B_{t}\right) d B_{t}$, where $B$ is the Brownian Motion, states that if $X_{t}=f\left(t, B_{t}\right)$ for some sufficiently regular function, it holds that $\mu=\frac{\partial f}{\partial t}+\frac{1}{2} \frac{\partial^{2} f}{\partial x^{2}}$ and $\sigma=\frac{\partial f}{\partial x}$. Then formula (2) follows by partial differentiation and an appropriate change of variables (see Theorem 7.1.7 and the final proof of this article). In fact, we derive a sort of discrete version of Ito's Lemma. Indeed, the Wiener Walk $W$ being recombining, it follows from elementary geometric considerations that any recombining process satisfying the stochastic difference equation (1) is a function $f\left(t, W_{t}\right)$ of time and the Wiener Walk. For such functions $f$ we derive formulae for $\mu$ and $\sigma$ which are analogous to those given by Ito's Lemma, now in terms of near-equalities and partial difference-quotients. In a sense the Main Theorem adds to this discrete Ito Lemma a converse, stating that a sufficiently regular function $f$ exists only if $\mu$ and $\sigma$ satisfy the partial differential equation (2).

The two ordinary differential equations used for the determination of the stochastic variables of the recombining processes $Y$ are obtained as infinitesimal approximations of ordinary difference equations, by the general nonstandard method of transition from the discrete to the continuous called stroboscopy. It is interesting to note that the differential equations are special cases of those obtained by Halim Doss [16] for continuous-time processes, with entirely different methods.

There is a new interest in the determination of expectations in view of the emergence of mathematical finance, for they may be interpreted as the price of options and other financial instruments. In [8] some steps are taken to apply the above-mentioned strategy in this context. The strategy may be seen as an extension of the method used in [7] and [10] to derive the Black Scholes price [9] of options for continuous-time pricing-models from the discrete pricing-model of Cox-Ross-Rubinstein [11]. In that case the process is the recombining - Discrete Geometric Brownian Motion, and the difference equations respectively differential equations reduce to Riemann-sums respectively Riemann-integrals.

We begin this article by recalling, in Section 2, some notions and properties with respect to discrete stochastic processes. In Section 2.1 we define some planar geometric notions, motivated by the regular, uniform behaviour of the trajectories of the Wiener Walk. In Section 2.2 we define formally discrete stochastic processes. In Section 2.3 we consider in particular the processes originating from stochastic difference equations and, within this category, in Section 2.4 those processes which are recombining. To the recombining processes we associate a geometric tool, the discrete surface, an infinitesimally fine network consisting of all points lying on the trajectories of the process. In Section 2.5 we consider the probability law of these processes, which is the Binomial 
Law; the latter is in the case of a sufficiently large number of steps infinitely close to the Normal Law, by the DeMoivre-Laplace central limit theorem. Section 2.6 recalls various notions of near-continuity and near-differentiability for discrete functions defined on infinitesimal grids, including functions of two variables. We indicate how the relation with standard continuous and (partially) differentiable functions is made through the notion of shadow. In Section 2.7 we recall the definition of near-equivalence of [22] and the criterion for near-equivalence for solutions of stochastic difference equations proved in [3].

The preliminary work of defining the principal notions and the setting being done, we are able to present the Main Theorem in Section 3. We give some examples and sketch the structure of the proofs of the sufficient and necessary parts.

In Section 4 we construct a recombining process $Y_{t}$ for every discrete stochastic process $X_{t}$ satisfying the stochastic difference equation (1), that we will call the associated recombining process. The construction is made by a form of vertical induction, starting from the central median trajectory of $X$. An important feature of the construction is that it modifies only the trend of the original process, maintaining its conditional variance.

In Section 5 we derive the two differential equations which permit to determine the limited part of the discrete surface $\widetilde{Y}$ up to an infinitesimal. Also, the regularity conditions of the Main Theorem permit to show that $\widetilde{Y}$ is limited and $S$-continuous for limited arguments, and as a consequence we obtain that nearly all trajectories of the associated recombining process are limited and $S$-continuous.

In Section 6 we analyze the error made when approximating the original stochastic process by the associated recombining process. Because the inductive method induces cumulative errors, in principle the approximation does not need to be very good. However, under the conditions of the Main Theorem the trend of the recombining process differs from the trend of the original process only infinitesimally for limited arguments. Knowing that the two processes have the same median trajectory, we are able to prove this by vertical induction, with the aid of the partial differential equation (2). Then a criterion on near-equivalence of [3] permits to conclude that the two processes are nearly-equivalent. As an application we show how to use the near-equivalence for the determination of expectations with respect to the original process. In this section we show also that the trend of the recombining process and the discrete surface associated to it have more regularity than already mentioned, for they have nonstandard properties of near-differentiability.

As already said, the relation between the trend and the conditional variance of the original process $X$, given by the partial differential equation (2), is necessary for our proof that the recombining process $Y$ resulting from the construction in Section 4 is nearly equivalent. In the last section we show that it is also sufficient: within the regularity conditions of the Main Theorem, the (shadows of) the trend and the conditional variance of a recombining process should satisfy (2). Indeed, the properties of near-differentiability of the associated discrete surface lead to some individual approximate partial difference equations for the trend and the conditional variance. Then their shadows satisfy the corresponding partial differential equations, and differentiating partially once again, together with some simplifications, leads to (2). We end with a corollary, in fact a reformulation of the Main Theorem, which relates the formulae in question to Ito's Lemma.

This article uses the axiomatic approach of Nonstandard Analysis I.S.T. of Nelson [21]. For an introduction and notations, we refer to [14] and [13], and for an introduction 
to stochastic processes in this setting to [22]. Some formulae contain the symbol $\varnothing$. This is a symbol of approximation, and is used much as the symbol $o(1)$ in asymptotic analysis. For example $x=\varnothing$ is an abbreviation of "there exists an infinitesimal number $\epsilon$ such that $x=\epsilon "$, while the exact value of $\epsilon$ does not have much importance. The use of this and similar symbols is formalized in [19].

We thank Marc and Francine Diener (University of Nice) and Eric Benoît (University of La Rochelle) for some useful discussions. We thank the referee for indicating an inconsistency in the proof of the Main Theorem.

\section{Notations and Definitions}

We introduce some notations, definitions and basic properties that will be used in the remaining sections.

\subsection{Geometric notions}

We let $\delta t>0, \delta t \simeq 0$ be an infinitesimal time-period. We write $\mathbb{T}=\{k \delta t: k \in \mathbb{N}\}$. Let $a, b \in \mathbb{T}, a \leq b$. We define the discrete interval $[a . . b] \subset \mathbb{T}$ by

$$
[a . . b]=\{t \in \mathbb{T}: a \leq t \leq b\} .
$$

We will also consider other discrete structures with points equally spaced at infinitesimal distance, and extend the notion of discrete interval to such structures. In particular we let $\delta x=2 \sqrt{\delta t}$. Then $\delta x \simeq 0$, but $\delta x / \delta t \simeq+\infty$. We write $\mathbb{X}=\{n \delta x: n \in \mathbb{Z}\}$. Also, if $\delta y>0, \delta y \simeq 0$, we write $\mathbb{Y}=\{m \delta y: m \in \mathbb{Z}\}$. To a discrete interval $[a . . b]$ may be associated a standard, continuous interval through the notion of shadow [14]. Indeed, if $t$ is a limited real number its shadow is the (unique) standard number ${ }^{\circ} t$ such that ${ }^{\circ} t \simeq t$. For instance, one shows that if $a$ and $b$ are both limited the shadow ${ }^{\circ}[a . . b]$ of $[a . . b]$ is the continuous interval $\left[{ }^{\circ} a,{ }^{\circ} b\right]$; also ${ }^{\circ} \mathbb{T}=[0, \infty),{ }^{\circ} \mathbb{X}=\mathbb{R}$ and ${ }^{\circ}(\mathbb{X} \times \mathbb{Y})=\mathbb{R}^{2}$. The notion of shadow serves also to associate to a discrete function with sufficient regularity a standard continuous function, see Section 2.6.

We define $m: \mathbb{T} \rightarrow \mathbb{R}$ by

$$
m(t)=\left\{\begin{array}{cc}
0 & t / \delta t \text { even } \\
\sqrt{\delta t} & t / \delta t \text { odd }
\end{array}\right.
$$

(see Figure 2).

Let $T \in \mathbb{T}$. The binomial cone $\mathcal{C}_{T} \subset \mathbb{R}^{+} \times \mathbb{R}$ is defined by

$$
\mathcal{C}_{T}=\left\{(t, x): t \in[0 . . T], x \in m(t)+\mathbb{X},|x| \leq \frac{t}{\sqrt{\delta t}}\right\} .
$$

See Figure 1; observe that ${ }^{\circ} \mathcal{C}_{T}=\left[0,{ }^{\circ} T\right] \times \mathbb{R}$.

\subsection{Stochastic processes}

We consider discrete stochastic processes $X$ in the sense of [22], indexed by [0..T], where $T \in \mathbb{T}$. They may be seen as a sequence $\left(X_{t}\right)_{t \in[0 . . T]}$ of stochastic variables 


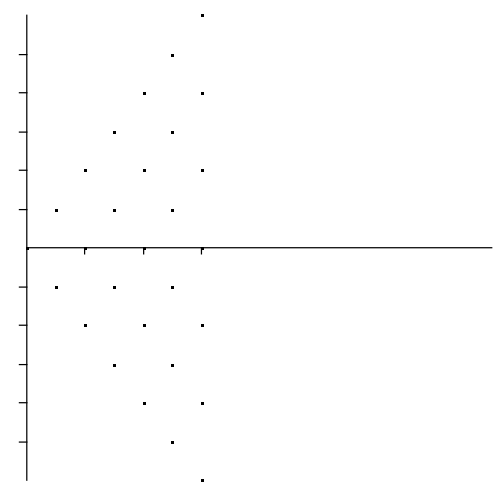

Figure 1. The binomial cone $\mathcal{C}_{T}$

defined on the same finite probability space $\left\langle\Omega, p r>\right.$, i.e. every $X_{t}$ is a function of $\Omega$ into $\mathbb{R}$. Let $\omega \in \Omega$. Then the function $\xi:[0 . . T] \rightarrow \mathbb{R}$, defined by

$$
\xi(t)=X_{t}(\omega),
$$

is called a trajectory of $X$. It is thus possible (but not necessary) to identify $\Omega$ with the set of trajectories of the process $X$. In this article we consider stochastic processes defined inductively, as solutions of stochastic difference equations. This means that an initial stochastic variable $X_{0}$ is given and an equation for its increments $\delta X_{t} \equiv X_{t+\delta t}-X_{t}$, for $t \in[0 . . T-\delta t]$. An important particular case is given by the Wiener Walk $W$. This process has constant initial condition $W_{0}=0$ and its increments $\delta W_{t}$ satisfy

$$
\delta W_{t}=\left\{\begin{array}{cl}
\sqrt{\delta t} & \text { probability } \frac{1}{2} \\
-\sqrt{\delta t} & \text { probability } \frac{1}{2}
\end{array}\right.
$$

Then every trajectory $\lambda$ is at every instant $t$ given by the partial sum

$$
\lambda(t)=\sum_{0 \leq s<t}(-1)^{\varepsilon(s)} \sqrt{\delta t},
$$

where $\varepsilon(s)=1$ or $\varepsilon(s)=0$. Note that $m(t)=\sum_{0 \leq s<t}(-1)^{s / \delta t} \sqrt{\delta t}$, so $m$ is a trajectory of the Wiener Walk. We call $m$ the median trajectory.

Let $\Lambda_{T}$ be the set of the $2^{T / \delta t}$ possible trajectories of the Wiener Walk. If we suppose that its increments are independent, it may be shown that all trajectories $\lambda$ are equiprobable with probability $\operatorname{pr}(\lambda)=\left(\frac{1}{2}\right)^{T / \delta t}$. Then $<\Lambda_{T}, p r>$ is a probability space. The binomial cone $\mathcal{C}_{T}$ is the union of all trajectories of the Wiener Walk.

\subsection{Stochastic difference equations}

Let $T \in \mathbb{T}, T>0$. We consider stochastic difference equations of the form

$$
\left\{\begin{array}{ll}
\delta X_{t} & =\mu\left(t, X_{t}\right) \delta t+\sigma\left(t, X_{t}\right) \delta W_{t} \\
X_{0} & =x_{0}
\end{array} \quad t \in[0 \cdots T-\delta t]\right.
$$




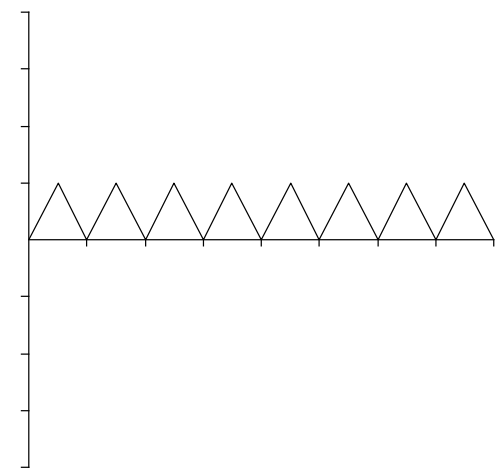

Figure 2. The median trajectory $m$ of the Wiener Walk.

with $\mu$ and $\sigma>0$ real functions of two variables and $x_{0} \in \mathbb{R}$. By (3) every trajectory $\lambda$ of the Wiener Walk defines a function $\xi \equiv \xi(\lambda):[0 . . T] \rightarrow \mathbb{R}$. Hence we may consider the solution $X$ of (3) to be the sequence of the partial sums $X_{t}=x_{0}+\sum_{0 \leq s<t} \delta X_{s}$, all defined on the probability space $\left\langle\Lambda_{T}, p r\right\rangle$. We suppose that at every $t \in[0 . . T-\delta t]$ the functions $\mu(t, \cdot)$ and $\sigma(t, \cdot)$ are defined at least on $X_{t}\left(\Lambda_{T}\right)$. The function $\mu$ is called the trend and the function $\sigma^{2}$ the conditional variance of the process.

\subsection{Recombining processes}

Let $X$ be a stochastic process satisfying the stochastic difference equation (3) and $t \in[0 . . T-\delta t]$. The upward movement $\delta X_{t}^{+}$is defined by

$$
\delta X_{t}^{+}=\mu\left(t, X_{t}\right)+\sigma\left(t, X_{t}\right) \sqrt{\delta t}
$$

and the downward movement $\delta X_{t}^{-}$is defined by

$$
\delta X_{t}^{-}=\mu\left(t, X_{t}\right)-\sigma\left(t, X_{t}\right) \sqrt{\delta t}
$$

We define $X_{t}^{+}=X_{t}+\delta X_{t}^{+}$and $X_{t}^{-}=X_{t}+\delta X_{t}^{-}$. For the stochastic variables resulting from two successive movements one defines in an evident way the notation $X_{t}^{++}, X_{t}^{+-}$, $X_{t}^{-+}$and $X_{t}^{--}$, where $t<T-\delta t$.

A process is said to be recombining if for every $t<T-\delta t$

$$
X_{t}^{+-}=X_{t}^{-+}
$$

Examples of recombining processes are the Wiener Walk and the Discrete Geometric Brownian Motion $S$ defined by $S_{0}=1$ and

$$
\delta S_{t}=\bar{\mu} S_{t} \delta t+\bar{\sigma} S_{t} \delta W_{t}
$$

with $\bar{\mu}, \bar{\sigma} \in \mathbb{R}, \bar{\sigma} \neq 0$.

To a recombining process $X$ one may associate a mapping, called discrete surface

$$
\widetilde{X}: \mathcal{C}_{T} \rightarrow \mathbb{R}
$$


defined by

$$
\widetilde{X}(t, x)=X_{t}(\lambda)
$$

for some $\lambda \in \Lambda$ such that $\lambda(t)=x$. The mapping is well-defined because recombination implies path-independence: if $\lambda^{\prime} \in \Lambda$ is such that $\lambda^{\prime}(t)=x$, one has $X_{t}\left(\lambda^{\prime}\right)=X_{t}(\lambda)$. The discrete surface associated to the Wiener Walk is the plane

$$
\widetilde{W}(t, x)=x,
$$

and for the Geometric Brownian Motion with $\bar{\mu}, \bar{\sigma}$ standard one has the approximation

$$
\widetilde{S}(t, x) \simeq \exp \left(\left(\bar{\mu}-\frac{1}{2} \bar{\sigma}^{2}\right) t+\bar{\sigma} x\right)
$$

valid for limited $t$ and $x$; see for instance [10][7]. In Section 5 we derive a general method to determine approximations of discrete surfaces.

Let $T \in \mathbb{T}, T>0$. Let $X$ be a recombining stochastic process indexed by $[0 \cdots T]$ and $\widetilde{X}$ be its associated discrete surface. Then we may associate to a function $f$ of $t$ and $X_{t}$ a function $\widetilde{f}: \mathcal{C}_{T} \rightarrow \mathbb{R}$ by defining $\widetilde{f}(t, x)=f(t, \widetilde{X}(t, x))$. For instance, to the trend $\mu$, respectively the conditional variance $\sigma^{2}$, of a recombining process we associate functions $\widetilde{\mu}$, respectively $\widetilde{\sigma}$, defined on $\mathcal{C}_{T}$, given by $\widetilde{\mu}(t, x)=\mu(t, \widetilde{X}(t, x))$ and $\widetilde{\sigma}(t, x)=\sigma(t, \widetilde{X}(t, x))$.

To a given discrete surface $\widetilde{X}: \mathcal{C}_{T} \rightarrow \mathbb{R}$ it is possible to associate a recombining stochastic process, satisfying a stochastic difference equation of the form (3), by defining

$$
\left\{\begin{array}{l}
\mu(t, \tilde{X}(t, x))=\frac{\widetilde{X}(t+\delta t, x+\sqrt{\delta t})+\widetilde{X}(t+\delta t, x-\sqrt{\delta t})-2 \widetilde{X}(t, x)}{\widetilde{X}(t+\delta t, x+\sqrt{\delta t})-\widetilde{X}(t+\delta t, x-\sqrt{\delta t})} \\
\sigma(t, \widetilde{X}(t, x))=\frac{2 \delta t}{2 \sqrt{\delta t}} \\
\left\{\begin{array}{l}
\delta X_{t}=\mu \\
X_{0}=\widetilde{X}(0,0) .
\end{array}\right.
\end{array}\right.
$$

With abuse of language we identify sometimes a recombining process with its discrete surface.

\subsection{Binomial distributions}

The probability distribution of a recombining process given by the stochastic difference equation (3) is binomial. Indeed, put for $(t, x) \in \mathcal{C}_{T}$

$$
\begin{aligned}
\nu_{t} & =\frac{t}{\delta t} \\
j_{t, x} & =\frac{t}{2 \delta t}+\frac{x}{\delta x} \\
b(t, x) & =\frac{1}{\delta x}\left(\begin{array}{c}
\nu_{t} \\
j_{t, x}
\end{array}\right) \cdot \frac{1}{2^{t / \delta t}}
\end{aligned}
$$

Then

$$
\operatorname{Pr}\left\{X_{t}=\tilde{X}(t, x)\right\}=\operatorname{Pr}\left\{W_{t}=x\right\}=\left(\begin{array}{c}
\nu_{t} \\
j_{t, x}
\end{array}\right) \cdot \frac{1}{2^{t / \delta t}}=b(t, x) \delta x
$$

Clearly

$$
\sum_{|x| \leq \frac{t}{\sqrt{\delta t}}} b(t, x) \delta x=1
$$


For appreciable $t$ and limited $x$ we have the Gaussian approximation

$$
b(t, x) \simeq \frac{e^{-\frac{x^{2}}{2 t}}}{\sqrt{2 \pi t}}
$$

For more details of this nonstandard version of the DeMoivre-Laplace central limit theorem we refer to [7].

\subsection{Near-continuity and near-differentiability}

Let $D \subset \mathbb{R}$ and $f: D \rightarrow \mathbb{R}$ be a function. The function $f$ is said to be $S$-continuous if for every $x, y \in D$

$$
x \simeq y \Rightarrow f(x) \simeq f(y) .
$$

The function $f$ is said to be of class $S^{0}$ if it is limited and $S$-continuous at least at the limited elements of $D$ [14]. Both continuous functions and discrete functions may be of class $S^{0}$. It may be shown that every standard everywhere defined continuous function is limited and $S$-continuous on the external set of limited numbers, hence is of class $S^{0}$, and also that to every discrete function $f$ defined on a near-interval $[a . . b]$ may be associated a standard continuous function ${ }^{\circ} f$, called shadow of $f$. If $a$ and $b$ are limited, one has ${ }^{\circ} f:\left[{ }^{\circ} a,{ }^{\circ} b\right] \rightarrow \mathbb{R}$, with $f(x) \simeq{ }^{\circ} f(x)$ for all $x \in[a . . b] \cap\left[{ }^{\circ} a,{ }^{\circ} b\right]$. If $a$ and $b$ are unlimited, its shadow ${ }^{\circ} f$ is defined on $\mathbb{R}$, and $f(x) \simeq{ }^{\circ} f(x)$ holds for all limited $x \in[a . . b]$.

One may extend these notions of near-continuity of discrete functions to higher order and to functions of two variables. For instance, let $\delta x>0, \delta x \simeq 0$. A function $f: \mathbb{X} \rightarrow \mathbb{R}$ is called of class $S^{1}$ if $f$ and $\delta f / \delta x$ are of class $S^{0}$, with $\delta f$ defined by $\delta f(x)=f(x+$ $\delta x)-f(x)$. The function $f$ is called of class $S^{2}$ if $f$ is of class $S^{1}$ and $\frac{\delta^{2} f}{\delta x^{2}}$ is of class $S^{0}$. On may show that the shadow of a function of class $S^{1}$ is of class $C^{1}$ and that the shadow of a function of class $S^{2}$ is of class $C^{2}$ [6]. The following definition concerns discrete functions of two variables.

Definition 2.1 Let $\delta x, \delta y>0, \delta x, \delta y \simeq 0$. Let $f: \mathbb{X} \times \mathbb{Y} \rightarrow \mathbb{R}$ and $D \subset \mathbb{X} \times \mathbb{Y}$. We write $\delta_{1} f=f(x+\delta x, y)-f(x, y), \delta_{2} f=f(x, y+\delta y)-f(x, y)$ and $\delta_{i j}^{2} f=\delta_{i}\left(\delta_{j} f\right)$ for $i, j \in\{1,2\}$. We say that

1) $f$ is of class $S^{0,0}$ on $D$ if $f$ is limited and $S$-continuous at every limited point $(x, y) \in D$.

2) $f$ is of class $S^{1,0}$ on $D$ if $f$ and $\frac{\delta_{1} f}{\delta x}$ are of class $S^{0,0}$ on $D$.

3) $f$ is of class $S^{0,1}$ on $D$ if $f$ and $\frac{\delta_{2} f}{\delta y}$ are of class $S^{0,0}$ on $D$.

4) $f$ is of class $S^{2,0}$ on $D$ if $f$ is of class $S^{1,0}$ and $\frac{\delta_{11}^{2} f}{\delta x^{2}}$ is of class $S^{0,0}$ on D. In an analogous way we define functions of class $S^{0,2}$.

5) Let $i, j \in\{1,2\}$. We say that $f$ is of class $S^{i, j}$ on $D$ if $f$ is of class $S^{i, 0}$ and $S^{0, j}$ on $D$.

For $i, j \in\{1,2\}$ the shadow of a function of class $S^{i, j}$ is a function of class $C^{i, j}$. For more details and proofs, see [6] and [15].

It is straightforward to extend the notion of class $S^{0}$ to discrete functions defined on irregular sets of points. For instance, let $f: \mathbb{X} \rightarrow \mathbb{R}$ be of class $S^{0}$. Let $g: f(\mathbb{X}) \rightarrow \mathbb{R}$. We say that $g$ is of class $S^{0}$ if $g(y)$ is limited for all limited $y \in f(\mathbb{X})$ and $g(y) \simeq h(z)$ 
whenever $y, z \in f(\mathbb{X})$ are limited with $y \simeq z$. Then $g \circ f$ is of class $S^{0}$ and ${ }^{\circ} g$ is defined at least on the shadow of the image by $f$ of the limited part of $\mathbb{X}$. More care is needed for near-differentiability. The function $g$ is said to be of class $S^{1}$ if $g$ is of class $S^{0}$ and there exists a function $h: f(\mathbb{X}) \rightarrow \mathbb{R}$ of class $S^{0}$ such that $\frac{g(z)-g(y)}{z-y} \simeq h(y)$ whenever $y, z \in f(\mathbb{X})$ are limited with $y \simeq z$. Then ${ }^{\circ} g$ is of class $C^{1}[14][6]$ and $\left({ }^{\circ} g\right)^{\prime}={ }^{\circ} h$. If $f$ is also of class $S^{1}$, we have the following "Chain Rule" for limited $x \in \mathbb{X}$ :

$$
\circ\left(\frac{g(f(x+\delta x))-g(f(x))}{\delta x}\right)=\left(\left({ }^{\circ} g\right)^{\prime} \circ{ }^{\circ} f\right)(x) \cdot\left({ }^{\circ} f\right)^{\prime}(x) .
$$

Indeed,

$$
\begin{gathered}
\frac{\delta(g \circ f)(x)}{\delta x}=\frac{g(f(x+\delta x))-g(f(x))}{\delta x} \\
=\frac{g(f(x)+\delta f(x))-g(f(x))}{\delta f(x)} \frac{\delta f(x)}{\delta x} \simeq h(f(x)) \frac{\delta f(x)}{\delta x},
\end{gathered}
$$

so $\frac{\delta(g \circ f)}{\delta x}$ is of class $S^{0}$. Hence $g \circ f$ is of class $S^{1}$. Then ${ }^{\circ}(g \circ f)$ is of class $C^{1}$, with ${ }^{\circ}\left(\frac{g(f(x+\delta x))-g(f(x))}{\delta x}\right)=\left({ }^{\circ} h \circ \circ f\right)(x) \cdot\left({ }^{\circ} f\right)^{\prime}(x)=\left(\left({ }^{\circ} g\right)^{\prime} \circ{ }^{\circ} f\right)(x) \cdot\left({ }^{\circ} f\right)^{\prime}(x)$ of class $C^{0}$.

We consider also near-continuity and near-differentiability of functions of two variables. In particular, let $f: \mathbb{X} \times \mathbb{Y} \rightarrow \mathbb{R}^{2}$ be such that $f(x, y)=(x, \phi(y))$, for some function $\phi: \mathbb{Y} \rightarrow \mathbb{R}$ of class $S^{0}$. Let $g: f(\mathbb{X} \times \mathbb{Y}) \rightarrow \mathbb{R}$. We say that $g$ is of class $S^{0,0}$ if $g(x, z)$ is limited for all limited $(x, z) \in f(\mathbb{X} \times \mathbb{Y})$ and $g\left(x_{1}, z_{1}\right) \simeq g\left(x_{2}, z_{2}\right)$ whenever $\left(x_{1}, z_{1}\right),\left(x_{2}, z_{2}\right) \in f(\mathbb{X} \times \mathbb{Y})$ are limited with $\left(x_{1}, z_{1}\right) \simeq\left(x_{2}, z_{2}\right)$; then ${ }^{\circ} g$ is of class $C^{0,0}$. The function $g$ is said to be of class $S^{0,1}$ if $g$ is of class $S^{0,0}$ and there exists a function $h$ of class $S^{0,0}$ such that $\frac{g(x, w)-g(x, z)}{w-z} \simeq h(x, z)$ whenever $z, w \in \phi(\mathbb{Y})$ are limited with $z \simeq w$. Then for limited $(x, z) \in \mathbb{X} \times \phi(\mathbb{Y})$ one has ${ }^{\circ}\left(\frac{g(x, w)-g(x, z)}{w-z}\right)=$ $\left(\frac{\partial^{\circ} g}{\partial^{\circ} \phi}\right)(x, z)$. If $\phi$ is also of class $S^{1}$, the "Chain Rule" for limited $(x, y) \in \mathbb{X} \times \mathbb{Y}$ takes the form

$$
\circ\left(\frac{g(x, \phi(y+\delta y))-g(x, \phi(y))}{\delta y}\right)=\left(\frac{\partial g}{\partial{ }^{\circ} \phi} \circ{ }^{\circ} \phi\right)(x, y) \cdot\left(\frac{\partial{ }^{\circ} \phi}{\partial y}\right)(x, y) .
$$

for limited $(x, y) \in \mathbb{X} \times \mathbb{Y}$.

In [22] Nelson defined a form of $S$-continuity also for real-valued functionals $F$ defined on sets of trajectories of stochastic processes, say, indexed by $[0 . . T]$, with $T \in \mathbb{T}$. Indeed, let $\Theta$ be such a set. Then $F: \Theta \rightarrow \mathbb{R}$ is uniformly $S$-continuous if $F(\alpha) \simeq F(\beta)$ for all trajectories $\alpha, \beta \in \Theta$ such that $\alpha(t) \simeq \beta(t)$ for all $t \in[0 . . T]$.

\subsection{Expectations, nearly-equivalent processes}

Let $\delta t>0$ and $T \in \mathbb{T}$. Let $X$ be a discrete stochastic process indexed by $[0 . . T]$, solution of the stochastic difference equation (3). Then $X$ is defined on the probability space $<\Lambda_{T}, p r>$. Let $\Xi$ be the set of all trajectories of $X$. Let $F: \Xi \rightarrow \mathbb{R}$. Then the expectation of $F$ is

$$
E F=\sum_{\lambda \in \Lambda_{T}} F(\xi(\lambda)) \operatorname{pr} \lambda
$$


Since there are $2^{T / \delta t}$ trajectories, this formula is of exponential complexity. If $F$ depends only on the final value of the trajectory $\xi(\lambda)$, and the process is recombining, one obtains a significant simplification. Indeed, formula (5) may be reduced to a binomial sum, in fact a Riemann-sum, i.e.

$$
E F=\sum_{|x| \leq T / \sqrt{\delta t}} b(T, x) \widetilde{F}(\widetilde{X}(T, x)) \delta x,
$$

with $\widetilde{F}: \operatorname{Im} X_{T} \rightarrow \mathbb{R}$ defined by $\widetilde{F}(\xi(\lambda)(T))=F(\xi(\lambda))$. If $T$ is limited and $F$ is limited and uniformly $S$-continuous, and also $\widetilde{X}(T, \cdot)$ is of class $S^{0}$, the above Riemann-sum is nearly-equal to the improper Riemann-integral

$$
E F \simeq \frac{1}{\sqrt{2 \pi T}} \int_{-\infty}^{+\infty} e^{-\frac{x^{2}}{2 T}} \cdot{ }^{\circ} \widetilde{F}\left({ }^{\circ} \widetilde{X}(T, x)\right) d x,
$$

see [5].

Definition 2.2 Let $\delta t>0, \delta t \simeq 0$ and $T \in \mathbb{T}$. Let $X$ and $Y$ be two discrete stochastic processes defined on the same probability space, indexed by $[0 . . T]$. Let $\Theta$ be the union of all trajectories of $X$ and $Y$. Then $X$ and $Y$ are said to be nearly-equivalent if for all limited and uniformly $S$-continuous functionals $F$ defined on $\Theta$

$$
E F(X) \simeq E F(Y) \text {. }
$$

In [3] (see also [4]) a sufficient condition on the trend and conditional variance is given for two stochastic difference equations to have nearly-equivalent solutions. We present a version of this result, which is adapted to our setting. First we recall the nonstandard probabilistic notion of nearly everywhere.

Let $\langle\Omega, p r\rangle$ be a finite probability space. An (internal or external) property $P$ is said to hold nearly everywhere if for all standard $a>0$ there exists an internal set $I \subset\{P(x)\}$ such that $\operatorname{Pr} I \geq 1-a$; it is not difficult to show that an internal property $P$ holds nearly everywhere if and only if $\operatorname{Pr}\{P(x)\} \simeq 1$. In [22] it is shown that, when the Wiener Walk is indexed by a discrete time-interval $[0 \cdots T]$, with limited $T$, nearly all trajectories are everywhere $S$-continuous.

Theorem 2.3 Let $\delta t>0, \delta t \simeq 0$ and $T \in \mathbb{T}$ be appreciable. Let $X$ and $Y$ be two discrete stochastic processes indexed on $[0 \cdot T]$. Suppose that $X$ satisfies the stochastic difference equation (3), where ${ }^{\circ} \mu$ is standard of class $C^{0,1}$ and ${ }^{\circ} \sigma>0$ is standard of class $C^{1,2}$, with $\partial^{\circ} \mu / \partial X$ bounded, and that $Y$ satisfies the stochastic difference equation

$$
\left\{\begin{array}{l}
\delta Y_{t}=\nu\left(t, Y_{t}\right) \delta t+\rho\left(t, Y_{t}\right) \delta W_{t} \\
Y_{0}=y_{0}
\end{array}\right.
$$

where ${ }^{\circ} \nu={ }^{\circ} \mu$ and ${ }^{\circ} \rho={ }^{\circ} \sigma$. If nearly all trajectories of one of the processes are everywhere limited, the processes $X$ and $Y$ are nearly-equivalent.

We note that although Benoît supposed ${ }^{\circ} \mu$ and ${ }^{\circ} \sigma$ to be of class $C^{\infty, \infty}$, in his proof he uses only that ${ }^{\circ} \mu$ is of class $C^{0,1}$ and ${ }^{\circ} \sigma$ is of class $C^{1,2}$. We will show in Section 5.3 that, if $\partial^{\circ} \sigma / \partial X$ is also bounded, nearly all trajectories of the recombining process $Y$ associated to the process $X$ are everywhere limited (hence also nearly all trajectories of the process $X$, by near-equivalence [22]). 


\section{The Main Theorem}

For convenience we consider stochastic processes indexed by a discrete interval $[0 \cdot T]$, with $T>0$ standard. I.e., we might suppose the final time $T$ as given, and assume then that the interval $[0, T]$ is divided into an unlimited number of infinitesimal time-steps of equal length $\delta t$.

Theorem 3.1 Let $T>0, x_{0} \in \mathbb{R}$ be standard. Let $\delta t>0, \delta t \simeq 0$ be such that $T / \delta t \in \mathbb{N}$. Let $\sigma:[0, T] \times \mathbb{R} \rightarrow \mathbb{R}$ be standard of class $C^{1,2}$, with $\sigma>0$ and $\frac{\partial \sigma}{\partial X}$ bounded.

1) Let $\mu:[0, T] \times \mathbb{R} \rightarrow \mathbb{R}$ be standard of class $C^{0,1}$. Let $X$ satisfy the stochastic difference equation (3). Assume $\frac{\partial \mu}{\partial X}$ is bounded, and

$$
\sigma \frac{\partial \mu}{\partial X}-\left(\frac{\partial}{\partial t}+\mu \frac{\partial}{\partial X}+\frac{\sigma^{2}}{2} \frac{\partial^{2}}{\partial X^{2}}\right) \sigma=0
$$

Then there exists a recombining process $Y$, which is nearly equivalent to $X$ on $[0 \cdots T]$ and satisfies a stochastic difference equation

$$
\left\{\begin{array}{l}
\delta Y_{t}=\nu\left(t, Y_{t}\right) \delta t+\sigma\left(t, Y_{t}\right) \delta W_{t} \\
Y_{0}=x_{0}
\end{array} \quad t \in[0 \cdot T-\delta t]\right.
$$

with $\nu:\left\{(t, \tilde{Y}(t, x)) \mid(t, x) \in \mathcal{C}_{T}\right\} \rightarrow \mathbb{R}$ of class $S^{0,1}$.

2) Let $Y$ be a recombining process, satisfying the stochastic difference equation (9), where $\nu:\left\{(t, \widetilde{Y}(t, x)) \mid(t, x) \in \mathcal{C}_{T}\right\} \rightarrow \mathbb{R}$ is of class $S^{0,1}$ and $\frac{\nu(t, \widetilde{Y}(t, x+\delta x))-\nu(t, \widetilde{Y}(t, x))}{\delta_{2} \widetilde{Y}(t, x)}$ is limited for $(t, x) \in \mathcal{C}_{T-\delta t}$, with $x<t / \sqrt{\delta t}$. Let $\mu={ }^{\circ} \nu$. Then (i) $X={ }^{\circ} \widetilde{Y}$ is well-defined as a function of $[0, T] \times \mathbb{R}$ into $\mathbb{R}$, (ii) $\mu: X([0, T] \times \mathbb{R}) \rightarrow \mathbb{R}$ is of class $C^{0,1}$ with $\frac{\partial \mu}{\partial X}$ bounded and (iii) $\mu$ and $\sigma$ satisfy (8).

The proof of the first part of the theorem involves the following steps. Firstly, we construct a recombining stochastic process $Y$ corresponding to the process $X$ with the same conditional variance, but with possibly modified trend $\nu$, that we will call the associated recombining process (Section 4). Secondly, we determine the discrete surface corresponding to the associated recombining process up to an infinitesimal, by solving two ordinary differential equations; we prove also that the surface is at least of class $S^{0,0}$ and obtain as a consequence that nearly all trajectories of the recombining process are of class $S^{0}$ (Section 5). Thirdly, we show that the shadow of the trend $\nu$ of the recombining process is equal to the trend $\mu$ of the original process, being even of class $S^{0,1}$. The two latter properties enable to apply Benoît's theorem on the near-equivalence of processes (Section 6).

The near-equality of the trend of the original process and the trend of the associated recombining process relies heavily on the partial differential equation (8). Indeed, one may show that the original process is in a sense "nearly recombining": all the terms of the Taylor expansion of $X_{t}^{+-}-X_{t}^{-+}$, i.e. the discrepancy between the result of an upward movement followed by a downward movement and the result of a downward movement followed by an upward movement, cancel up to order $\delta t^{3 / 2}$, the coefficient of the latter term being $2\left(\sigma \frac{\partial \mu}{\partial X}-\frac{\partial \sigma}{\partial t}-\mu \frac{\partial \sigma}{\partial X}-\frac{\sigma^{2}}{2} \frac{\partial^{2} \sigma}{\partial X^{2}}\right)$. This will also imply that the difference between $X$ and $Y$ is sufficiently small for near-equivalence; see the remark following the proof of the first part of the Main Theorem in Section 6. 
The proof of the second part of the theorem is contained in Section 7. Theorem 7.1.1, 7.1.2, 7.1.4 and 7.1.6 state that the functions involved have appropriate regularity. Theorem 7.1.3 states some partial difference equations for the discrete surface $\widetilde{Y}$ associated to the process $Y$ and Theorem 7.1.5 some partial differential equations for its shadow $X \equiv{ }^{\circ} \widetilde{Y}$. Appropriate partial differentiation of these partial differential equations (Theorem 7.1.7) and a change of variables lead to the partial differential equation (8). We will also show that some of the equations in question have an interpretation in terms of Ito's formula.

We end this section with some examples.

\section{Examples.}

1) The trend and the conditional standard deviation of the Wiener Walk and the Discrete Geometric Brownian Motion (4) satisfy the partial differential equation (8). Indeed, in the case of the Wiener Walk $\delta W_{t}=1 \cdot \delta W_{t}$ all partial derivatives are zero, and in the case of the Discrete Geometric Brownian Motion $\delta S_{t}=\bar{\mu} S_{t} \delta t+\bar{\sigma} S_{t} \delta W_{t}$ we have

$$
\bar{\sigma} S_{t} \frac{\partial \bar{\mu} S}{\partial S}-\frac{\partial \bar{\sigma} S}{\partial t}-\bar{\mu} S \frac{\partial \bar{\sigma} S}{\partial S}-\frac{\bar{\sigma}^{2} S^{2}}{2} \frac{\partial^{2} \bar{\sigma} S}{\partial S^{2}}=\overline{\sigma \mu} S-\overline{\mu \sigma} S=0
$$

2) It is easily verified that the Geometric Brownian Motion with time-dependent "drift", i.e. a process originating from equations of the form

$$
\delta S_{t}=\mu(t) S_{t} \delta t+\bar{\sigma} S_{t} \delta W_{t} \quad S_{0}=s_{0}
$$

satisfies the conditions of Part 1 of the Main Theorem, if $\mu$ is a standard and continuous real function and $\bar{\sigma}$ and $s_{0}$ are standard positive real numbers. This is also true for processes satisfying a stochastic diference equation of the form

$$
\delta X_{t}=\frac{\sigma^{\prime}(t)}{\sigma(t)} X_{t} \delta t+\sigma(t) \delta W_{t} \quad X_{0}=x_{0},
$$

where $\sigma$ is standard, positive and continuously differentiable, and $x_{0} \in \mathbb{R}$ is standard.

3) Let $x_{0}, a$ and $C$ be standard positive numbers with $a \neq 1$. The trend and the conditional variance of the processes given by

$$
\delta X_{t}=\left\{\begin{array}{l}
\left(\frac{a}{2} X_{t}^{2 a-1}+C X_{t}^{a}\right) \delta t+X_{t}^{a} \delta W_{t} \\
X_{0}=x_{0}
\end{array}\right.
$$

satisfy (8). The shadow of the associated discrete surface $\widetilde{Y}$ may be given in closed form. Indeed, with the aid of Theorem 5.3 below, it may be seen that ${ }^{\circ} \tilde{Y}(t, x)=V_{t}(x)$, where $V_{t}$ is solution of the differential equation

$$
\left\{\begin{array}{l}
\frac{d V_{t}}{d x}=\left(V_{t}(x)\right)^{a} \\
V(0)=U(t)
\end{array}\right.
$$

with $U(t)$ given by

$$
\left\{\begin{array}{c}
\frac{d U}{d s}=C U^{a}(s) \\
U(0)=x_{0}
\end{array}\right.
$$

Then ${ }^{\circ} \tilde{Y}(t, x)=\left(x_{0}^{1-a}+(1-a)(x+C t)\right)^{\frac{1}{1-a}}$. Notice however that it may very well not be defined everywhere, depending on the value of $a$, while also the growth and/or the 
differentiability conditions for the trend and the conditional variance of the Main Theorem are not satisfied in general. Such problems could be overcome by introducing appropriate reflecting barriers (which should respect a property of "near-recombination") or stopping times (then the process would be "nearly recombining" only until stopped). Still, the process obtained by these restrictions would not satisfy the conditions of differentiability of the Main Theorem, so it needs further investigation to conclude that they are nearlyequivalent to a recombining process.

\section{The associated recombining process}

Let $X$ be a process satisfying the stochastic difference equation (3) for $t \in[0 \cdot \cdot T-\delta t]$, with $T \in \mathbb{T}, T>0$.

We construct a recombining stochastic process $Y$ corresponding to the process $X$ with the same conditional variance, but with possibly modified trend $\nu$. In fact we define $Y$ through its associated discrete surface $\widetilde{Y}$. We start by defining $\widetilde{Y}$ along the median trajectory $m$ of the Wiener Walk, and continue the construction by induction in $x$.

Definition 4.1 Let $\delta t>0$ and $T \in \mathbb{T}, T>0$. Let the process $X$ satisfy the stochastic difference equation (3) for $t \in[0 \cdot T-\delta t]$. The associated recombining process $Y$ is defined by

1) (Initial values) We define

$$
\begin{cases}\tilde{Y}(t, m(t))=X_{t}(m) & t \in[0 \cdot T] \\ \widetilde{\sigma}(t, m(t))=\sigma\left(t, X_{t}(m)\right) & t \in[0 \cdot T-\delta t] \\ \widetilde{\nu}(t, m(t))=\mu\left(t, X_{t}(m)\right) & t \in[0 \cdot T-\delta t] .\end{cases}
$$

2) (Induction step)

a) (Positive $x$ ) Let $x>0$ be a multiple of $\sqrt{\delta t}$ and suppose $\widetilde{Y}(t, y), \widetilde{\sigma}(t, y)$ and $\widetilde{\mu}(t, y)$ are defined for all $(t, y) \in \mathcal{C}_{T}$ such that $0 \leq y \leq x$ and $y \sqrt{\delta t} \leq t \leq T$ (in the case of $\widetilde{Y}$ ) and $y \sqrt{\delta t} \leq t<T$ (in the case of $\widetilde{\sigma}$ and $\widetilde{\nu})$. We define for $(t, x+\sqrt{\delta t}) \in \mathcal{C}_{T}$

$$
\widetilde{Y}(t, x+\sqrt{\delta t})=\widetilde{Y}(t-\delta t, x)+\widetilde{\nu}(t-\delta t, x) \delta t+\widetilde{\sigma}(t-\delta t, x) \sqrt{\delta t} .
$$

and for $(t, x+\sqrt{\delta t}) \in \mathcal{C}_{T}$ such that $t<T$

$$
\left\{\begin{array}{l}
\widetilde{\sigma}(t, x+\sqrt{\delta t})=\sigma(t, \widetilde{Y}(t, x+\sqrt{\delta t})) . \\
\widetilde{\nu}(t, x+\sqrt{\delta t})=\widetilde{\nu}(t, x-\sqrt{\delta t})+\frac{\widetilde{\sigma}(t, x+\sqrt{\delta t})-2 \widetilde{\sigma}(t-\delta t, x)+\widetilde{\sigma}(t, x-\sqrt{\delta t})}{\sqrt{\delta t}} .
\end{array}\right.
$$

b) (Negative $x$ ) Let $x<0$ be a multiple of $\sqrt{\delta t}$ and suppose $\tilde{Y}(t, y), \widetilde{\sigma}(t, y)$ and $\widetilde{\nu}(t, y)$ are defined for all $(t, y) \in \mathcal{C}_{T}$ such that $x \leq y \leq 0$ and $y \sqrt{\delta t} \leq t \leq T$ (in the case of $\widetilde{Y}$ ) and $y \sqrt{\delta t} \leq t<T$ (in the case of $\widetilde{\sigma}$ and $\widetilde{\nu}$ ). We define for $(t, x-\sqrt{\delta t}) \in \mathcal{C}_{T}$

$$
\widetilde{Y}(t, x-\sqrt{\delta t})=\widetilde{Y}(t-\delta t, x)+\widetilde{\nu}(t-\delta t, x) \delta t-\widetilde{\sigma}(t-\delta t, x) \sqrt{\delta t} .
$$

and for $(t, x-\sqrt{\delta t}) \in \mathcal{C}_{T}$ such that $t<T$

$$
\left\{\begin{array}{l}
\widetilde{\sigma}(t, x-\sqrt{\delta t})=\sigma(t, \widetilde{Y}(t, x-\sqrt{\delta t})) . \\
\widetilde{\nu}(t, x-\sqrt{\delta t})=\widetilde{\nu}(t, x+\sqrt{\delta t})-\frac{\widetilde{\sigma}(t, x-\sqrt{\delta t})-2 \widetilde{\sigma}(t-\delta t, x)+\widetilde{\sigma}(t, x-\sqrt{\delta t})}{\sqrt{\delta t}} .
\end{array}\right.
$$


Observe that in the induction step both the positive and the negative case reduce to the same formula of $\widetilde{\nu}(t, x+\sqrt{\delta t})$ in terms of $\widetilde{\nu}(t, x-\sqrt{\delta t})$, i.e.

$$
\widetilde{\nu}(t, x+\sqrt{\delta t})=\widetilde{\nu}(t, x-\sqrt{\delta t})+\frac{\widetilde{\sigma}(t, x+\sqrt{\delta t})-2 \widetilde{\sigma}(t-\delta t, x)+\widetilde{\sigma}(t, x-\sqrt{\delta t})}{\sqrt{\delta t}} .
$$

Next proposition verifies that the process $Y$ is recombining indeed.

Proposition 4.2 Let $\delta t>0$ and $T \in \mathbb{T}, T>0$. Let $X$ be a process satisfying the stochastic difference equation (3) for $t \in[0 \cdots T-\delta t]$. The process $Y$ given by Definition 4.1 is recombining.

Proof. Let $(t-\delta t, x) \in \mathcal{C}_{T}$ such that still $(t+\delta t, x) \in \mathcal{C}_{T}$. Then, applying (11),

$$
\begin{aligned}
& \widetilde{Y}(t-\delta t, x)^{+-}-\widetilde{Y}(t-\delta t, x)^{-+}= \\
= & \widetilde{\nu}(t-\delta t, x) \delta t+\widetilde{\sigma}(t-\delta t, x) \sqrt{\delta t}+\widetilde{\nu}(t, x+\sqrt{\delta t}) \delta t-\widetilde{\sigma}(t, x+\sqrt{\delta t}) \sqrt{\delta t}+ \\
& -(\widetilde{\nu}(t-\delta t, x) \delta t-\widetilde{\sigma}(t-\delta t, x) \sqrt{\delta t}+\widetilde{\nu}(t, x-\sqrt{\delta t}) \delta t+\widetilde{\sigma}(t, x-\sqrt{\delta t}) \sqrt{\delta t}) \\
= & (\widetilde{\nu}(t, x+\sqrt{\delta t})-\widetilde{\nu}(t, x-\sqrt{\delta t})) \delta t+ \\
& -(\widetilde{\sigma}(t, x+\sqrt{\delta t}) \sqrt{\delta t}-2 \widetilde{\sigma}(t-\delta t, x) \sqrt{\delta t}+\widetilde{\sigma}(t, x-\sqrt{\delta t})) \sqrt{\delta t} \\
= & \frac{\widetilde{\sigma}(t, x+\sqrt{\delta t})-2 \widetilde{\sigma}(t-\delta t, x)+\widetilde{\sigma}(t, x-\sqrt{\delta t})}{\sqrt{\delta t}} \delta t+ \\
= & -(\widetilde{\sigma}(t, x+\sqrt{\delta t}) \sqrt{\delta t}-2 \widetilde{\sigma}(t-\delta t, x) \sqrt{\delta t}+\widetilde{\sigma}(t, x-\sqrt{\delta t})) \sqrt{\delta t} \\
= & .
\end{aligned}
$$

\section{Properties of the discrete surface}

On rather mild conditions on $\mu$ and $\sigma$ the discrete surface $\widetilde{Y}$ has a shadow, which can be determined by solving successively two ordinary differential equations.

We start, in the general context of processes $X$ which are solutions of stochastic difference equations, by deriving approximations of $X_{t}^{+-}-X_{t}$ and $X^{+}-X^{-}$.

Proposition 5.1 Let $\delta t>0$ and $T \in \mathbb{T}, T>0$. Let $X$ be a process satisfying the stochastic difference equation (3), where $\mu$ is of class $S^{0,0}$ and $\sigma$ is standard of class $C^{1,1}$. Let $t \leq T-2 \delta$ t and $\xi$ be a trajectory such that $\xi(t)$ is limited and which has an upward movement at time $t$ and a downward movement at time $t+\delta t$. Then

$$
\frac{\xi(t+2 \delta t)-\xi(t)}{2 \delta t} \simeq \mu(t, \xi(t))-\frac{1}{2} \sigma(t, \xi(t)) \frac{\partial \sigma(t, \xi(t))}{\partial X} .
$$

Proof. One has

$$
\begin{gathered}
\frac{\xi(t+2 \delta t)-\xi(t)}{2 \delta t}= \\
\frac{\mu(t, \xi(t))+\mu(t+\delta t, \xi(t+\delta t))}{2}-\frac{\sigma(t+\delta t, \xi(t+\delta t))-\sigma(t, \xi(t))}{2 \sqrt{\delta t}} .
\end{gathered}
$$


Because $\mu(t, \xi(t))$ and $\sigma(t, \xi(t))$ are limited, $\xi(t+\delta t)$ is also limited. So

$$
\mu(t+\delta t, \xi(t+\delta t)) \simeq \mu(t, \xi(t)),
$$

because $\mu$ is $S$-continuous. Hence $(\mu(t, \xi(t))+\mu(t+\delta t, \xi(t+\delta t))) / 2 \simeq \mu(t, \xi(t))$.

Also,

$$
\begin{aligned}
& \frac{\sigma(t+\delta t, \xi(t+\delta t))-\sigma(t, \xi(t))}{2 \sqrt{\delta t}}= \\
& \frac{1}{2 \sqrt{\delta t}} \cdot[\sigma(t+\delta t, \xi(t)+\mu(t, \xi(t)) \delta t+\sigma(t, \xi(t) \sqrt{\delta t})+ \\
& -\sigma(t, \xi(t)+\mu(t, \xi(t)) \delta t+\sigma(t, \xi(t)) \sqrt{\delta t})]+ \\
& +\frac{\sigma(t, \xi(t)+\mu(t, \xi(t)) \delta t+\sigma(t, \xi(t)) \sqrt{\delta t})-\sigma(t, \xi(t))}{\mu(t, \xi(t)) \delta t+\sigma(t, \xi(t)) \sqrt{\delta t}} \times \\
& \times \frac{\mu(t, \xi(t)) \delta t+\sigma(t, \xi(t)) \sqrt{\delta t}}{2 \sqrt{\delta t}} \\
& =\frac{\frac{\partial \sigma}{\partial t}(t, \xi(t)) \delta t+\varnothing \delta t}{2 \sqrt{\delta t}}+\left(\frac{\partial \sigma}{\partial X}(t, \xi(t))+\varnothing(\mu(t, \xi(t)) \delta t+\sigma(t, \xi(t)) \sqrt{\delta t})\right) \times \\
& \times \frac{\sigma(t, \xi(t)) \sqrt{\delta t}+\varnothing \sqrt{\delta t}}{2 \sqrt{\delta t}} \\
& =\frac{1}{2} \sigma(t, \xi(t)) \frac{\partial \sigma}{\partial X}(t, \xi(t))+\varnothing
\end{aligned}
$$

Combining, we derive (12).

Proposition 5.2 Let $\delta t>0$ and $T \in \mathbb{T}, T>0$. Let $X$ be a process satisfying the stochastic difference equation (3). Let $t<T$ and $\xi$ be a trajectory. Then

$$
\begin{gathered}
\frac{\xi(t)+\mu(t, \xi(t)) \delta t+\sigma(t, \xi(t)) \sqrt{\delta t}-(\xi(t)+\mu(t, \xi(t)) \delta t-\sigma(t, \xi(t)) \sqrt{\delta t})}{\delta x} \\
=\sigma(t, \xi(t)) .
\end{gathered}
$$

If in addition $\sigma$ is of class $C^{0,0}$ and $t$ and $\xi(t)$ are limited,

$$
\begin{array}{r}
\frac{\xi(t)+\mu(t, \xi(t)) \delta t+\sigma(t, \xi(t)) \sqrt{\delta t}-(\xi(t)+\mu(t, \xi(t)) \delta t-\sigma(t, \xi(t)) \sqrt{\delta t})}{\delta x} \\
\simeq \sigma(t+\delta t, \xi(t)+\mu(t, \xi(t)) \delta t-\sigma(t, \xi(t)) \sqrt{\delta t})
\end{array}
$$

We omit the obvious proof.

Theorem 5.3 Let $\delta t>0$ and $T \in \mathbb{T}, T>0$ be appreciable. Let $X$ be a process satisfying the stochastic difference equation (3), where $\mu$ is standard of class $C^{0,1}$ and $\sigma$ is standard of class $C^{1,2}$. Assume $\frac{\partial \mu}{\partial X}$ and $\frac{\partial \sigma}{\partial X}$ are bounded. Let $(t, x) \in \mathcal{C}_{T}$ be such that $x$ is limited. 
Let $Y$ be the associated recombining process. Let $U$ be the solution of the differential equation

$$
\left\{\begin{array}{l}
\frac{d U}{d s}=\mu(s, U)-\frac{1}{2} \sigma(s, U) \frac{\partial \sigma(s, U)}{\partial U} \\
U(0)=x_{0} .
\end{array}\right.
$$

Then $\widetilde{Y}(t, x) \simeq V_{t}(x)$, where $V_{t}$ is the solution of the differential equation

$$
\left\{\begin{array}{l}
\frac{d V_{t}}{d x}=\sigma\left(t, V_{t}(x)\right) \\
V(0)=U(t)
\end{array}\right.
$$

Moreover, the discrete surface $\widetilde{Y}$ is of class $S^{0,0}$, with shadow of class $C^{0,0}$.

Proof. Let $(t, x) \in \mathcal{C}_{T}$ be such that $x$ is limited. As a consequence of path-independence of the process $Y$, we may determine $\widetilde{Y}(t, x)$, by first following a horizontal path on $\mathcal{C}_{T}$, and then a vertical path. More precisely, we calculate

$$
\tilde{Y}(t, x)=\tilde{Y}(t, x)-\tilde{Y}(t, 0)+\tilde{Y}(t, 0)-\tilde{Y}(0,0)+\tilde{Y}(0,0)
$$

if $t / \delta t$ is even and

$$
\tilde{Y}(t, x)=\tilde{Y}(t, x)-\tilde{Y}(t, \sqrt{\delta t})+\tilde{Y}(t, \sqrt{\delta t})-\tilde{Y}(\sqrt{\delta t}, \sqrt{\delta t})+\tilde{Y}(\sqrt{\delta t}, \sqrt{\delta t})
$$

if $t / \delta t$ is odd. The calculations are very similar and we treat only the first case. 5.1

We consider first differences in horizontal direction. Let $s \leq T-2 \delta t$. By Proposition

$$
\frac{\tilde{Y}(s+2 \delta t, 0)-\tilde{Y}(s, 0)}{2 \delta t} \simeq \mu(s, \widetilde{Y}(s, 0))-\frac{1}{2} \sigma(s, \widetilde{Y}(s, 0)) \frac{\partial \sigma}{\partial \widetilde{Y}}(s, \widetilde{Y}(s, 0)) .
$$

The second member of this difference equation is of class $S^{0,1}$. The second member of the differential equation $\frac{d U}{d s}=\mu(s, U)-\frac{1}{2} \sigma(s, U) \frac{\partial \sigma(s, U)}{\partial U}$ is of class $C^{0,1}$, and because $\frac{\partial \mu(s, U)}{\partial U}$ and $\frac{\partial \sigma(s, U)}{\partial U}$ are bounded, it has existence and uniqueness of solutions. By the Stroboscopy Theorem [26] and the Strong Short Shadow Lemma of [14], the solutions of (16) with initial condition $\widetilde{Y}(0,0)=x_{0}$ and of (13) are limited and infinitely close on $[0 \cdot T]$.

Second, we consider differences in vertical direction. Let $x$ be such that $(t, x) \in \mathcal{C}_{T}$. We will suppose that $x>0$, the case $x<0$ is analogous. By Proposition 5.2

$$
\frac{\tilde{Y}(t, x+\delta x)-\tilde{Y}(t, x)}{\delta x}=\sigma(t-\delta t, \tilde{Y}(t-\delta t, x+\sqrt{\delta t})) .
$$

The second member of this difference equation is of class $S^{1,2}$. The second member of the differential equation $\frac{d V_{o_{t}}}{d x}=\sigma\left({ }^{\circ} t, V_{\circ}\right)$ is of class $C^{1,2}$, hence it has existence and uniqueness of solutions. By the Stroboscopy Theorem [26] and the Strong Short Shadow Lemma of [14] the solutions of (16) with initial condition $\widetilde{Y}(t, 0)=U(t)$ and of $\frac{d V_{\circ} t}{d x}=\sigma\left({ }^{\circ} t, V_{\circ} t(x)\right), V_{{ }^{\circ} t}(0)=U\left({ }^{\circ} t\right)$ are limited and infinitely close on $[0 \cdot x]$. Again by the Strong Short Shadow Lemma the solutions of (14) and of $\frac{d V_{o_{t}}}{d x}=\sigma\left({ }^{\circ} t, V_{\circ} t(x)\right)$, $V_{\bullet} t(0)=U\left({ }^{\circ} t\right)$ are limited and infinitely close on $[0 \cdot x]$. We conclude from (15) that $\widetilde{Y}(t, x) \simeq V_{t}(x)$. 
To prove the remaining part of the theorem, let $(t, x),(\tau, \xi) \in \mathcal{C}_{T}$ with $\tau \simeq t$ and $\xi \simeq x$. Because $U$ is of class $S^{0}$, one has $U(\tau) \simeq U(t)$. It follows from the Strong Short Shadow Lemma that the solutions of $\frac{d V_{\tau}}{d x}=\sigma\left(\tau, V_{\tau}(x)\right), V_{\tau}(0)=U(\tau)$ and of (14) are limited and infinitely close on $[0 \cdots x]$. Because $V_{\tau}$ is of class $S^{0}$, one has $\tilde{Y}(\tau, \xi) \simeq$ $V_{\tau}(\xi) \simeq V_{t}(x) \simeq \widetilde{Y}(t, x)$. We conclude that the function $\tilde{Y}$ is of class $S^{0,0}$. Then ${ }^{\circ} \widetilde{Y}$ is of class $C^{0,0}$.

Note that one uses the trend and conditional variance of the original process $X$ in determining the infinitesimal approximation of the discrete surface corresponding to the associated recombining process. In fact, one uses the trend of the process $X$ only along the trajectory corresponding to the median trajectory of the Wiener walk, in the differential equation (13), since the differential equation (14) is defined in terms of the time and the conditional variance.

For recombining processes satisfying the conditions of the second part of the Main Theorem one has a theorem similar to Theorem 5.3.

Theorem 5.4 Let $T>0$ be standard. Let $\delta t>0, \delta t \simeq 0$ be such that $T / \delta t \in \mathbb{N}$. Let $Y$ be a recombining process satisfying the stochastic difference equation (9), where $\nu$ : $\{(t, \tilde{Y}(t, x))$

$\left.\mid(t, x) \in \mathcal{C}_{T}\right\} \rightarrow \mathbb{R}$ is of class $S^{0,1}$ and is such that $\frac{\nu(t, \widetilde{Y}(t, x+\delta x))-\nu(t, \widetilde{Y}(t, x))}{\widetilde{Y}(t, x+\delta x)-\widetilde{Y}(t, x)}$ is limited for $(t, x) \in \mathcal{C}_{T-\delta t}, x<t / \sqrt{\delta t}$, and where $\sigma$ is standard of class $C^{1,2}$, with $\frac{\partial \sigma}{\partial Y}$ bounded. Let $\mu:[0, T] \times \mathbb{R} \rightarrow \mathbb{R}$ be the shadow of $\nu$. Let $(t, x) \in \mathcal{C}_{T}$, with $x$ limited. Let $U$ be the solution of the differential equation

$$
\left\{\begin{array}{l}
\frac{d U}{d s}=\mu(s, U)-\frac{1}{2} \sigma(s, U) \frac{\partial \sigma(s, U)}{\partial U} \\
U(0)=x_{0} .
\end{array}\right.
$$

Then $\tilde{Y}(t, x) \simeq V_{t}(x)$, where $V_{t}$ is the solution of the differential equation

$$
\left\{\begin{array}{l}
\frac{d V_{t}}{d x}=\sigma\left(t, V_{t}(x)\right) \\
V(0)=U(t) .
\end{array}\right.
$$

Moreover, the discrete surface $\widetilde{Y}$ is of class $S^{0,0}$, with shadow of class $C^{0,0}$.

Proof. We note that $\mu$ is of class $C^{0,1}$, with $\frac{\partial \mu}{\partial Y}$ bounded. Then the proof is entirely similar to the proof of the previous theorem.

Under the conditions of Theorem 5.3 we obtain as a corollary that nearly all trajectories of the recombining process associated to a solution of (3) are of class $S^{0}$.

Corollary 5.5 Let $T>0$ be standard. Let $\delta t>0, \delta t \simeq 0$ be such that $T / \delta t \in \mathbb{N}$. Let $X$ be a process satisfying the stochastic difference equation (3), where $\mu$ is standard of class $C^{0,1}$ and $\sigma$ is standard of class $C^{1,2}$. Assume $\frac{\partial \mu}{\partial X}$ and $\frac{\partial \sigma}{\partial X}$ are bounded. Let $Y$ be the associated recombining process.

1) Let $\lambda$ be a S-continuous trajectory of Wiener Walk. Let $\eta(\lambda)$ be the corresponding trajectory of $Y$. Then $\eta(\lambda)$ is $S$-continuous.

2) Nearly all trajectories of $Y$ are of class $S^{0}$.

3) Nearly all trajectories of $X$ are of class $S^{0}$. 
Proof. 1. Let $\lambda$ be a S-continuous trajectory, and $t_{1}, t_{2} \leq T$ be limited with $t_{1} \simeq t_{2}$. Then $\lambda\left(t_{1}\right) \simeq \lambda\left(t_{2}\right)$. As $\widetilde{Y}$ is of class $S^{00}$, one has

$$
\eta(\lambda)\left(t_{1}\right)=\tilde{Y}\left(t_{1}, \lambda\left(t_{1}\right)\right) \simeq \widetilde{Y}\left(t_{2}, \lambda\left(t_{2}\right)\right)=\eta(\lambda)\left(t_{2}\right) .
$$

2. The property is a consequence of Part 1 , and the fact that nearly all trajectories of the Wiener Walk indexed by a discrete time-interval of limited length are everywhere $S$-continuous.

3. Clearly, if a trajectory $\xi$ is everywhere limited, all trajectories $\eta$ with $\eta(t) \simeq \xi(t)$ for all $t \in[0 \cdot T]$ are everywhere limited. Then it follows from [22] that, if some process has the property that nearly all trajectories are everywhere limited, it is shared by every nearly-equivalent process. Hence by Part 2 nearly all trajectories of $X$ are everywhere limited.

\section{Near-equivalence of a process and the associated recombining process}

Let $X$ be a stochastic process and $Y$ be the associated recombining process. In this section we prove that under the conditions of the Main Theorem they are nearly equivalent. As a consequence we obtain an approximation method for stochastic variables related to the original process.

In the lemma below and in its proof, whenever a function is defined at $(t, \widetilde{Y}(t, x))$, we omit the argument.

Lemma 6.1 Let $\delta t>0$ and $T \in \mathbb{T}, T>0$ be appreciable. Let $X$ be a process satisfying the stochastic difference equation (3), where $\mu$ is standard of class $C^{0,1}$ and $\sigma$ is standard of class $C^{1,2}$. Let $Y$ be the associated recombining process. Let $(t, x) \in \mathcal{C}_{T}$ such that $t<T$ and $x$ is limited.

1) One has

$$
\frac{\widetilde{\mu}(t+\delta t, x+\sqrt{\delta t})-\widetilde{\mu}(t+\delta t, x-\sqrt{\delta t})}{2 \sqrt{\delta t}} \simeq \sigma \frac{\partial \mu}{\partial X} .
$$

2) Assume that $\nu(t, \tilde{Y}(t, x))$ limited. Then

$$
\frac{\widetilde{\nu}(t+\delta t, x+\sqrt{\delta t})-\widetilde{\nu}(t+\delta t, x-\sqrt{\delta t})}{2 \sqrt{\delta t}} \simeq \frac{\partial \sigma}{\partial t}+\nu \frac{\partial \sigma}{\partial X}+\frac{\sigma^{2}}{2} \frac{\partial^{2} \sigma}{\partial X^{2}} .
$$

3) Assume that $\nu(t, \tilde{Y}(t, x))$ limited and (8) holds. Then

$$
\begin{aligned}
\frac{\widetilde{\nu}(t+\delta t, x+\sqrt{\delta t})-\widetilde{\mu}(t+\delta t, x+\sqrt{\delta t})-(\widetilde{\nu}(t+\delta t, x}{2 \sqrt{\delta t}} & \sqrt{\delta t})-\widetilde{\mu}(t+\delta t, x-\sqrt{\delta t})) \\
& \simeq(\widetilde{\nu}(t, x)-\widetilde{\mu}(t, x)) \frac{\partial \sigma}{\partial X} .
\end{aligned}
$$


Proof. By Theorem 5.3 one has that $\widetilde{Y}(t, x)$ is limited and $S$-continuous in both of its variables.

1) Because $\mu$ is standard and of class $C^{0,1}$ and $\tilde{Y}(t+\delta t, x+\sqrt{\delta t})-\tilde{Y}(t+\delta t, x-$ $\sqrt{\delta t})=2 \sigma \sqrt{\delta t}$, one has

$$
\begin{aligned}
\frac{\widetilde{\mu}(t+\delta t, x+\sqrt{\delta t})-\widetilde{\mu}(t+\delta t, x-\sqrt{\delta t})}{2 \sqrt{\delta t}} & \\
\frac{\mu(t+\delta t, \widetilde{Y}(t+\delta t, x+\sqrt{\delta t}))-\mu(t+\delta t, \tilde{Y}(t+\delta t, x-\sqrt{\delta t}))}{\widetilde{Y}(t+\delta t, x+\sqrt{\delta t})-\widetilde{Y}(t+\delta t, x-\sqrt{\delta t})} & \simeq \\
\sigma \frac{\partial \mu}{\partial X}(t+\delta t, \widetilde{Y}(t+\delta t, x-\sqrt{\delta t})) & \simeq \sigma \frac{\partial \mu}{\partial X}
\end{aligned}
$$

2) We apply a Taylor-expansion up to order $\delta t$ to $\sigma$. Noting that the terms of order 0 and $\sqrt{\delta t}$ in the enumerator of the fraction below cancel, we obtain

$$
\begin{aligned}
& \frac{\widetilde{\nu}(t+\delta t, x+\sqrt{\delta t})-\widetilde{\nu}(t+\delta t, x-\sqrt{\delta t})}{2 \sqrt{\delta t}}= \\
& \frac{\sigma(t+\delta t, \widetilde{Y}(t+\delta t, x+\sqrt{\delta t}))-2 \sigma+\sigma(t+\delta t, \widetilde{Y}(t+\delta t, x-\sqrt{\delta t}))}{2 \delta t}= \\
& \frac{\sigma(t+\delta t, \tilde{Y}(t, x)+\nu \delta t+\sigma \sqrt{\delta t})-2 \sigma+\sigma(t+\delta t, \tilde{Y}(t, x)+\nu \delta t-\sigma \sqrt{\delta t})}{2 \delta t} \simeq \\
& \frac{\partial \sigma}{\partial t}+\nu \frac{\partial \sigma}{\partial X}+\frac{\sigma^{2}}{2} \frac{\partial^{2} \sigma}{\partial X^{2}} .
\end{aligned}
$$

3) By Parts 1 and 2, one has

$$
\begin{gathered}
\frac{\widetilde{\nu}(t+\delta t, x+\sqrt{\delta t})-\widetilde{\mu}(t+\delta t, x+\sqrt{\delta t})-(\widetilde{\nu}(t+\delta t, x-\sqrt{\delta t})-\widetilde{\mu}(t+\delta t, x-\sqrt{\delta t}))}{2 \sqrt{\delta t}}= \\
\frac{\widetilde{\nu}(t+\delta t, x+\sqrt{\delta t})-\widetilde{\nu}(t+\delta t, x-\sqrt{\delta t})}{2 \sqrt{\delta t}}-\frac{\widetilde{\mu}(t+\delta t, x+\sqrt{\delta t})-\widetilde{\mu}(t+\delta t, x-\sqrt{\delta t})}{2 \sqrt{\delta t}} \simeq \\
\frac{\partial \sigma}{\partial t}+\nu \frac{\partial \sigma}{\partial X}+\frac{\sigma^{2}}{2} \frac{\partial^{2} \sigma}{\partial X^{2}}-\sigma \frac{\partial \mu}{\partial X} .
\end{gathered}
$$

Now it follows from (8) that

$$
\frac{\partial \sigma}{\partial t}+\frac{\sigma^{2}}{2} \frac{\partial^{2} \sigma}{\partial X^{2}}-\sigma \frac{\partial \mu}{\partial X}=-\mu \frac{\partial \sigma}{\partial X} .
$$

Combining, we derive Lemma 6.1.3.

Next proposition states that the shadow of the modified trend $\nu$ is the original trend $\mu$ of the process $X$. Lemma 6.1.3 suggests a proof by stroboscopy (see also the remark on the role of equation (8) after the proof of the first part of the Main Theorem), but complications arise from the fact that the functions of the difference equation are defined 
at different points. Instead we apply the principle of nested induction from [6], which implies that a sequence which has a infinitesimal initial condition, and is such that from the hypothesis that at some step it has a limited value, one derives that at the next step it has in fact an infinitesimal value, remains infinitesimal for all limited arguments.

Proposition 6.2 Under the conditions of Theorem 3.1.1 one has ${ }^{\circ} \nu=\mu$.

Proof. Let $(t, x) \in \mathcal{C}_{T}$ be such that $t<T$ and $x$ is limited. We prove the proposition for $x \geq \sqrt{\delta t}$. The case $x \leq 0$ is analogous.

Put

$$
\left\{\begin{array}{l}
\widetilde{d}(t, x)=\widetilde{\nu}(t, x)-\widetilde{\mu}(t, x) \\
\widetilde{\epsilon}(t, x)=\frac{\widetilde{d}(t+\delta t, x+\sqrt{\delta t})-\widetilde{d}(t+\delta t, x-\sqrt{\delta t})}{2 \sqrt{\delta t}}-\widetilde{d}(t, x) \frac{\partial \sigma}{\partial X}(t, \widetilde{Y}(t, x)),
\end{array}\right.
$$

and

$$
\left\{\begin{array}{l}
D(x)=\max \left\{|\widetilde{d}(t, y)| \mid(t, y) \in \mathcal{C}_{T}, 0 \leq y \leq x\right\} \\
\epsilon(x)=\max \left\{|\widetilde{\epsilon}(t, y)| \mid(t, y) \in \mathcal{C}_{T}, 0 \leq y \leq x\right\}
\end{array}\right.
$$

Further, let

$$
S=\max \left\{\left|\frac{\partial \sigma}{\partial X}(t, \tilde{Y}(t, x))\right|(t, x) \in \mathcal{C}_{T-\delta t}\right\} .
$$

Assume $D(x)$ is limited. Then $\widetilde{\nu}(t, \widetilde{Y}(t, y))-\widetilde{\mu}(t, \widetilde{Y}(t, y))$ is limited for all $(t, y) \in \mathcal{C}_{T}$, with $0 \leq y \leq x$. Now $\tilde{Y}(t, y)$ is limited for all $(t, y) \in \mathcal{C}_{T}$ with $0 \leq y \leq x$, hence also $\widetilde{\mu}(t, \widetilde{Y}(t, y))$, and a fortiori $\widetilde{\nu}(t, \widetilde{Y}(t, y)$. Then Lemma 6.1 .3 implies that $\widetilde{\epsilon}(t, y) \simeq 0$ for all $(t, y) \in \mathcal{C}_{T}$ with $0 \leq y \leq x$, hence also $\epsilon(x) \simeq 0$. It follows from the identity

$$
\widetilde{d}(t+\delta t, x+\sqrt{\delta t})=\widetilde{d}(t+\delta t, x-\sqrt{\delta t})+2 \widetilde{d}(t, x) \frac{\partial \sigma}{\partial X}(t, \tilde{Y}(t, x)) \sqrt{\delta t}+2 \widetilde{\epsilon}(t, x) \sqrt{\delta t}
$$

that

$$
\begin{aligned}
\mid \widetilde{d}(t+\delta t, x+\sqrt{\delta t})) \mid & \leq D(x-\sqrt{\delta t})+2 D(x) S \sqrt{\delta t}+2 \epsilon(x) \sqrt{\delta t} \\
& \leq D(x)(1+2 S \sqrt{\delta t})+2 \epsilon(x) \sqrt{\delta t} .
\end{aligned}
$$

Because $D(x) \leq D(x)(1+2 S \sqrt{\delta t})+2 \epsilon(x) \sqrt{\delta t}$ and

$$
\left.D(x+\sqrt{\delta t})=\left(\max D(x), \max \{|\widetilde{d}(t, y)| \mid(t, y)) \in \mathcal{C}_{T}, y=x+\sqrt{\delta t}\right\}\right),
$$

also

$$
D(x+\sqrt{\delta t}) \leq D(x)(1+2 S \sqrt{\delta t})+2 \epsilon(x) \sqrt{\delta t} .
$$

Note that $D(\sqrt{\delta t})=0$, and that $D(x+\sqrt{\delta t}) \leq \Delta(x+\sqrt{\delta t})$, where $\Delta$ is the solution of the linear difference equation with constant coefficients

$$
\left\{\begin{array}{l}
\Delta(y+\sqrt{\delta t})=\Delta(y)(1+2 S \sqrt{\delta t})+2 \epsilon(x) \sqrt{\delta t} \\
\Delta(\sqrt{\delta t})=0
\end{array}\right.
$$

By the Stroboscopy Theorem and the Strong Short Shadow Lemma, the solution of this equation is infinitely close to the solution of

$$
\left\{\begin{array}{l}
\frac{d Z}{d y}=2 S Z \\
Z(0)=0
\end{array}\right.
$$


which is $Z \equiv 0$. Hence

$$
0 \leq D(x+\sqrt{\delta t}) \leq \Delta(x+\sqrt{\delta t}) \simeq Z(x+\sqrt{\delta t})=0 .
$$

So the hypothesis that $D(x)$ is limited implies that $D(x+\sqrt{\delta t}) \simeq 0$. By nested induction we conclude that $D(x)$ is limited for all limited $x \geq 0$. This implies that $\widetilde{\nu}(t, x) \simeq$ $\widetilde{\mu}(t, x)$ for all $(t, x) \in \mathcal{C}_{T}$ such that $x \geq \sqrt{\delta t}$ is limited. As already said, one obtains the analogous result for $x \leq 0$. We conclude that ${ }^{\circ} \nu=\mu$.

Proposition 6.3 Under the conditions of Theorem 3.1.1 the function $\nu:\{(t, \tilde{Y}(t, x))$ $\left.\mid(t, x) \in \mathcal{C}_{T}\right\} \rightarrow \mathbb{R}$ is of class $S^{0,1}$.

Proof. Because ${ }^{\circ} \nu=\mu$, the function $\nu$ is of class $S^{0,0}$. Let $(t, x) \in \mathcal{C}_{T}$ be such that $x$ is limited. We show that

$$
\frac{\nu(t, \widetilde{Y}(t, x+\delta x))-\nu(t, \widetilde{Y}(t, x))}{\widetilde{Y}(t, x+\delta x)-\widetilde{Y}(t, x)} \simeq \frac{\partial \mu}{\partial X}(t, \widetilde{Y}(t, x)) .
$$

Noting that $\widetilde{Y}, \nu, \mu, \frac{\partial \sigma}{\partial X}$ and $\frac{\partial \mu}{\partial X}$ are all of class $S^{0,0}$, it follows from Lemma 6.1.2 that

$$
\begin{aligned}
\frac{\nu(t, \tilde{Y}(t, x+\delta x))-\nu(t, \tilde{Y}(t, x))}{\delta x} & \simeq \\
(\nu(t, \tilde{Y}(t, x))-\mu(t, \tilde{Y}(t, x)) & \frac{\partial \sigma}{\partial X}(t, \tilde{Y}(t, x))+\sigma(t, \tilde{Y}(t, x)) \frac{\partial \mu}{\partial X}(t, \tilde{Y}(t, x)) .
\end{aligned}
$$

Because ${ }^{\circ} \nu=\mu$, and $\frac{\partial \sigma}{\partial X}(t, \widetilde{Y}(t, x))$ is limited, it follows that

$$
\frac{\nu(t, \widetilde{Y}(t, x+\delta x))-\nu(t, \widetilde{Y}(t, x))}{\widetilde{Y}(t, x+\delta x)-\widetilde{Y}(t, x)} \frac{\widetilde{Y}(t, x+\delta x)-\widetilde{Y}(t, x)}{\delta x} \simeq \sigma(t, \tilde{Y}(t, x)) \frac{\partial \mu}{\partial X}(t, \tilde{Y}(t, x)) .
$$

Then the fact that $(\tilde{Y}(t, x+\delta x)-\tilde{Y}(t, x)) / \delta x \simeq \sigma(t, \tilde{Y}(t, x))$ and $\sigma(t, \tilde{Y}(t, x))$ is appreciable implies (18). Because $\frac{\partial \mu}{\partial X}$ is of class $S^{0,0}$, also

$$
(\nu(t, \tilde{Y}(t, x+\delta x))-\nu(t, \tilde{Y}(t, x))) /(\widetilde{Y}(t, x+\delta x)-\tilde{Y}(t, x))
$$

is of class $S^{0,0}$. Hence $\nu$ is of class $S^{0,1}$.

Proof of the first part of the Main Theorem: By Proposition 6.2 one has ${ }^{\circ} \nu=\mu$. By Theorem 2.3 the processes $X$ and $Y$ are nearly-equivalent. By Proposition 6.3 the function $\nu:\left\{(t, \tilde{Y}(t, x)) \mid(t, x) \in \mathcal{C}_{T}\right\} \rightarrow \mathbb{R}$ is of class $S^{0,1}$.

We comment here the role of the partial differential equation $\sigma \frac{\partial \mu}{\partial X}-\frac{\partial \sigma}{\partial t}-\mu \frac{\partial \sigma}{\partial X}-$ $\frac{\sigma^{2}}{2} \frac{\partial^{2} \sigma}{\partial X^{2}}=0$ in the proof of the first part of the Main Theorem. Notice that it is only used in the proof of Lemma 6.1.3. The equation ensures that, when approximating the original process $X$ by the recombining process $Y$, the accumulation of the error in the difference $\nu-\mu$ of their respective trends by the inductive procedure of Definition 4.1 is infinitesimal. This follows from the fact that the trends are equal on the median trajectory $m$, and that their difference quotients in vertical direction are nearly equal. Indeed, now 
we know that $\nu$ is limited, if we suppose that ${ }^{\circ} \nu=\mu$ up to $x$, we infer from Lemma 6.1.2 that

$$
\frac{\widetilde{\nu}(t+\delta t, x+\sqrt{\delta t})-\widetilde{\nu}(t+\delta t, x-\sqrt{\delta t})}{2 \sqrt{\delta t}} \simeq \frac{\partial \sigma}{\partial t}+\mu \frac{\partial \sigma}{\partial X}+\frac{\sigma^{2}}{2} \frac{\partial^{2} \sigma}{\partial X^{2}} .
$$

Together with the near-equality

$$
\frac{\widetilde{\mu}(t+\delta t, x+\sqrt{\delta t})-\widetilde{\mu}(t+\delta t, x-\sqrt{\delta t})}{2 \sqrt{\delta t}} \simeq \sigma \frac{\partial \mu}{\partial X}
$$

of Lemma 6.1.1, one obtains that

$$
\begin{aligned}
\frac{\widetilde{\nu}(t+\delta t, x+\sqrt{\delta t})-\widetilde{\nu}(t+\delta t, x-\sqrt{\delta t})}{2 \sqrt{\delta t}} & -\frac{\widetilde{\mu}(t+\delta t, x+\sqrt{\delta t})-\widetilde{\mu}(t+\delta t, x-\sqrt{\delta t})}{2 \sqrt{\delta t}} \\
& \simeq \frac{\partial \sigma}{\partial t}+\mu \frac{\partial \sigma}{\partial X}+\frac{\sigma^{2}}{2} \frac{\partial^{2} \sigma}{\partial X^{2}}-\sigma \frac{\partial \mu}{\partial X}=0 .
\end{aligned}
$$

Next theorem states how the first part of the Main Theorem can be used to obtain an infinitesimal approximation of the expectations of functions $F$ of the stochastic variables of a process $X$, when it is nearly-equivalent to a recombining process.

Theorem 6.4 Let $X$ be a process with trend $\mu$ and conditional variance $\sigma^{2}$, solution of the stochastic difference equation (3) and satisfying the conditions of the first part of the Main Theorem. Let $F: \mathbb{R} \rightarrow \mathbb{R}$ be of class $S^{0}$ and bounded by some standard number. Assume $X$ is indexed by $[0 . . T]$, where $T \in \mathbb{T}, T>0$ is appreciable. Then for all appreciable $t \in[0 \cdots T]$

$$
E F\left(X_{t}\right) \simeq \frac{1}{\sqrt{2 \pi t}} \int_{-\infty}^{+\infty} \exp \left(-\frac{x^{2}}{2 t}\right){ }^{\circ} F(Z(t, x)) d x
$$

with $Z(t, x)=V_{t}(x)$, where $V_{t}$ is the solution of the initial value problem (14) with the initial condition $U(t)$ given by the initial value problem (13).

Proof. Let $Y$ be the recombining process given by Definition 4.1. Let $\Gamma$ be set of trajectories of $X$ and $\Delta$ be the set of trajectories of $Y$. Define $\Phi: \Gamma \cup \Delta \rightarrow \mathbb{R}$ by

$$
\Phi(\xi)=F(\xi(t))
$$

Let $\xi_{1}, \xi_{2} \in \Gamma \cup \Delta$ be two $S$-continuous trajectories such that $\xi_{1}(t) \simeq \xi_{2}(t)$ for all $t \in[0 . . T]$. Then

$$
\Phi\left(\xi_{1}\right)=F\left(\xi_{1}(t)\right) \simeq F\left(\xi_{2}(t)\right)=\Phi\left(\xi_{2}\right) .
$$

Now $\Phi$ is clearly limited. Also, it is uniformly $S$-continuous for nearly all elements of $\Gamma \cup \Delta$. Indeed, firstly, nearly all trajectories of $Y$ are $S$-continuous by Corollary 5.5.2, and nearly all trajectories of $X$ are $S$-continuous by Corollary 5.5.3. By [22], this suffices to conclude that $E F\left(X_{t}\right)=E \Phi(X) \simeq E \Phi(Y)=E F\left(Y_{t}\right)$. Then by formula (7)

$$
E F\left(X_{t}\right) \simeq \frac{1}{\sqrt{2 \pi t}} \int_{-\infty}^{+\infty} \exp \left(-\frac{x^{2}}{2 t}\right){ }^{\circ} F\left({ }^{\circ} \tilde{Y}(t, x)\right) d x
$$


Put $Z={ }^{\circ} \tilde{Y}$. It follows from Theorem 5.3 and the Transfer Principle that $Z(t, \cdot)=$ ${ }^{\circ} \tilde{Y}(t, \cdot)=V_{t}(\cdot)$, where $V_{t}$ is the solution of the initial value problem (14), and initial condition $U(t)$ given by (13).

The final proposition of this section states that the discrete surface associated to the recombining process has more regularity than already stated in Theorem 5.3.

Proposition 6.5 Under the conditions of Theorem 3.1.1 the function $\tilde{Y}: \mathcal{C}_{T} \rightarrow \mathbb{R}$ is of class $S^{1,2}$.

Proof. The function $\tilde{Y}: \mathcal{C}_{T} \rightarrow \mathbb{R}$ is of class $S^{0,0}$ by Theorem 5.3. Let $(t, x) \in \mathcal{C}_{T}$ be such that $x$ is limited and also $(t+2 \delta t, x) \in \mathcal{C}_{T}$. By Proposition 5.1 we have

$$
\frac{\tilde{Y}(t+2 \delta t, x)-\tilde{Y}(t, x)}{2 \delta t} \simeq \nu(t, \tilde{Y}(t, x))-\frac{1}{2} \sigma(t, \tilde{Y}(t, x)) \frac{\partial \sigma(t, \tilde{Y}(t, x))}{\partial X}
$$

Because $\nu(t, \tilde{Y}(t, x))-\frac{1}{2} \sigma(t, \tilde{Y}(t, x)) \frac{\partial \sigma(t, \tilde{Y}(t, x))}{\partial X}$ is of class $S^{0,0}$ (as a function of $t$ and $x)$, the function $\widetilde{Y}$ is of class $S^{1,0}$. Furthermore, by Proposition 5.2 we have

$$
\frac{\tilde{Y}(t, x+\delta x)-\tilde{Y}(t, x)}{\delta x}=\sigma(t-\delta t, \tilde{Y}(t-\delta t, x+\sqrt{\delta t}))
$$

Then the fact that $\sigma$ is of class $S^{0,0}$ implies that $\tilde{Y}$ is of class $S^{0,1}$. Further

$$
\begin{aligned}
& \frac{\frac{\delta_{2} \widetilde{Y}(t, x+\delta x)}{\delta x}-\frac{\delta_{2} \widetilde{Y}(t, x)}{\delta x}}{\delta x}= \\
& \quad \frac{\sigma(t-\delta t, \tilde{Y}(t-\delta t, x+3 \sqrt{\delta t}))-\sigma(t-\delta t, \tilde{Y}(t-\delta t, x+\sqrt{\delta t}))}{\widetilde{Y}(t-\delta t, x+3 \sqrt{\delta t})-\tilde{Y}(t-\delta t, x+\sqrt{\delta t})} \times \\
& \frac{\tilde{Y}(t-\delta t, x+3 \sqrt{\delta t})-\tilde{Y}(t-\delta t, x+\sqrt{\delta t})}{\delta x} \simeq \frac{\partial \sigma(t, \tilde{Y}(t, x))}{\partial X} \cdot \sigma(t, \tilde{Y}(t, x)) .
\end{aligned}
$$

Then the fact that $\frac{\partial \sigma}{\partial X} \cdot \sigma$ is of class $S^{0,0}$ implies that $\frac{\delta_{2} \widetilde{Y}(t, x)}{\delta x}$ is of class $S^{0,1}$. This means that $\tilde{Y}$ is of class $S^{0,2}$ [15]. Combining, we conclude that $\tilde{Y}$ is of class $S^{1,2}$.

\section{Partial differential equations associated to recombining processes.}

This final section contains the proof of the second part of the Main Theorem. The main task is to derive the macroscopic characterization of recombining processes, given by the partial differential equation (8), from the microscopic characterization of recombination, defined in terms of two successive increments. As already shown, a recombining process defines a discrete surface. The conditions of Theorem 3.1.2 imply that its trend and its conditional standard deviation have sufficient regularity to obtain equation (8) through a process of transforming partial difference-quotients of this discrete surface into the corresponding partial differential quotients. As a corollary we obtain a discrete version of Ito's formula [23][3]. 
Let $T \in \mathbb{T}, T>0$ be standard and let $\delta t>0, \delta t \simeq 0$ be such that $T / \delta t \in \mathbb{N}$. Let $Y$ be a recombining process indexed by $[0 \cdot T]$ and $\tilde{Y}$ be the associated discrete surface. Assume $\tilde{Y}$ is of class $S^{0,0}$, so its shadow $X \equiv{ }^{\circ} \widetilde{Y}$ is well-defined. Let $f$ be a function of two variables. For $(t, x) \in \mathcal{C}_{T}$ we write as usual $\widetilde{f}(t, x)=f(t, \tilde{Y}(t, x))$. For $(t, x) \in$ ${ }^{\circ} \mathcal{C}_{T}=[0, T] \times \mathbb{R}$ we write $\widehat{f}(t, x)=f(t, X(t, x))$.

Theorem 7.1 Let $T>0$ be standard. Let $\delta t>0, \delta t \simeq 0$ be such that $T / \delta t \in \mathbb{N}$. Let $Y$ be a recombining process satisfying the conditions of the second part of the Main Theorem. Let $\widetilde{Y}$ be the associated discrete surface. We put $X={ }^{\circ} \widetilde{Y}$ and $\mu={ }^{\circ} \nu$.

1) The discrete surface $\widetilde{Y}$ is of class $S^{1,2}$.

2) The discrete functions $\widetilde{\sigma}$ and $\widetilde{\nu}$ are of class $S^{0,1}$.

3) The function $\tilde{Y}:[0 \cdot T] \times \mathbb{R} \rightarrow \mathbb{R}$ satisfies

a) $\frac{\delta_{1} \widetilde{Y}}{2 \delta t} \simeq \widetilde{\nu}(t, x)-\frac{1}{2} \frac{\delta_{2} \widetilde{\sigma}}{\delta x}(t, x)$.

b) $\frac{\delta_{2} \widetilde{Y}}{\delta x} \simeq \widetilde{\sigma}(t, x)$.

c) $\frac{\delta_{2}^{2} \tilde{Y}}{\delta x^{2}} \simeq \frac{\delta_{2} \widetilde{\sigma}}{\delta x}(t, x)$.

d) $\frac{\delta_{1} \widetilde{Y}}{2 \delta t}+\frac{1}{2} \frac{\delta_{2}^{2} \widetilde{Y}}{\delta x^{2}} \simeq \widetilde{\nu}(t, x)$.

4) One has $\widehat{\mu}={ }^{\circ} \widetilde{\nu}$, and $\widehat{\mu}$ is defined and of class $C^{0,1}$ on $[0, T] \times \mathbb{R} \rightarrow \mathbb{R}$.

5) The function $X:[0, T] \times \mathbb{R} \rightarrow \mathbb{R}$ satisfies

a) $\frac{\partial X}{\partial t}=\widehat{\mu}(t, x)-\frac{1}{2} \frac{\partial \widehat{\sigma}(t, x)}{\partial x}$.

b) $\frac{\partial X}{\partial x}=\widehat{\sigma}(t, x)$.

c) $\frac{\partial^{2} X}{\partial x^{2}}=\frac{\partial \widehat{\sigma}(t, x)}{\partial x}$.

d) $\frac{\partial X}{\partial t}+\frac{1}{2} \frac{\partial X}{\partial x^{2}}=\widehat{\mu}(t, x)$.

6) The function $X$ is of class $C^{1,3}$.

7) $\frac{\partial \widehat{\mu}(t, x)}{\partial x}-\frac{\partial \widehat{\sigma}(t, x)}{\partial t}-\frac{1}{2} \frac{\partial^{2} \widehat{\sigma}(t, x)}{\partial x^{2}}=0$.

\section{Proof.}

1) The proof is entirely analogous to the proof of Proposition 6.5, applying Theorem 5.4 instead of Theorem 5.3.

2) We show first that $\widetilde{\sigma}$ is of class $S^{0,1}$. The fact that $\widetilde{\sigma}$ is of class $S^{0,0}$ follows from the formula $\widetilde{\sigma}(t, x)=\sigma(t, \widetilde{Y}(t, x))$ and the fact that $\widetilde{Y}$ is of class $S^{0,0}$. Moreover, using that $\sigma$ is of class $S^{0,1}$,

$$
\begin{aligned}
\frac{\delta_{2} \widetilde{\sigma}}{\delta x}(t, x) & =\frac{\sigma(t, \tilde{Y}(t, x+\delta x))-\sigma(t, \tilde{Y}(t, x))}{\widetilde{Y}(t, x+\delta x)-\widetilde{Y}(t, x)} \frac{\tilde{Y}(t, x+\delta x)-\tilde{Y}(t, x)}{\delta x} \\
& \simeq \frac{\partial \sigma(t, \tilde{Y}(t, x))}{\partial X} \widetilde{\sigma}(t, x) .
\end{aligned}
$$

Hence $\widetilde{\sigma}$ is also of class $S^{0,1}$. In analogous way one shows that $\widetilde{\nu}$ is of class $S^{0,1}$.

3) a) It follows from Proposition 5.1 and Part 2 that

$$
\frac{\delta_{1} \widetilde{Y}(t, x)}{2 \delta t} \simeq \nu(t, \tilde{Y}(t, x))-\frac{1}{2} \sigma(t, \tilde{Y}(t, x)) \frac{\partial \sigma(t, \widetilde{Y}(t, x))}{\partial X}=\widetilde{\nu}(t, x)-\frac{1}{2} \frac{\delta_{2} \widetilde{\sigma}}{\delta x}(t, x) \text {. }
$$


b) It follows from Proposition 5.2 and the fact that $\sigma$ is of class $S^{0,0}$ that

$$
\frac{\delta_{2} \tilde{Y}(t, x)}{\delta x}=\sigma(t-\delta t, \tilde{Y}(t-\delta t, x+\sqrt{\delta t})) \simeq \sigma(t, \tilde{Y}(t, x))=\tilde{\sigma}(t, x) .
$$
$S^{0,1}$.

c) The near-equality follows from Part $3 \mathrm{~b}$ and the fact that $\frac{\delta_{2}}{\partial x}$ and $\widetilde{\sigma}$ are both of class

d) The near-equality follows from Part $3 \mathrm{a}$ and Part $3 \mathrm{c}$.

4) Note that for $(t, x) \in \mathcal{C}_{T-\delta t}$, with limited $x$,

$$
\widehat{\mu}(t, x)=\mu(t, X(t, x)) \simeq \mu(t, \widetilde{Y}(t, x)) \simeq \nu(t, \widetilde{Y}(t, x))=\widetilde{\nu}(t, x) .
$$

So $\widehat{\mu}={ }^{\circ} \widetilde{\nu}$. By Part 2 the function $\widetilde{\nu}$ is of class $S^{0,1}$. Hence $\widehat{\mu}:[0, T] \times \mathbb{R} \rightarrow \mathbb{R}$ is of class $C^{0,1}$.

5) Since $\tilde{Y}$ is of class $S^{1,2}$ its shadow $X$ is at least of class $C^{1,2}$ and satisfies $\frac{\partial X}{\partial t}=$ $\circ \frac{\delta_{1} Y}{\delta t}, \frac{\partial X}{\partial x}=\circ \frac{\delta_{2} Y}{\delta x}$ and $\frac{\partial^{2} X}{\partial x^{2}}=\circ \frac{\delta_{2}^{2} \widetilde{Y}}{\delta x^{2}}$. Then the formulae follow from Part 3, using the equality $\frac{\partial \widehat{\sigma}(t, x)}{\partial x}=\circ\left(\frac{\delta_{2} \widetilde{\sigma}(t, x)}{\delta x}\right)$.

6) Because $\frac{\partial X}{\partial x}=\widehat{\sigma}(t, x)=\sigma(t, X(t, x))$ and $\sigma$ is of class $C^{0,2}$, and $X$ is already known to be of class $C^{0,2}$, the function $X$ is in fact of class $C^{0,3}$. Hence $X$ is of class $C^{1,3}$.

7) The formula will be obtained by differentiating the equality of Part 5a with respect to $x$. Observe that all terms of the formula are at least of class $C^{0,1}$. Indeed, $\frac{\partial X}{\partial t}$ is of class $C^{0,3}, \frac{\partial \widehat{\sigma}(t, x)}{\partial x}$ is of class $C^{1,1}$ and $\widehat{\mu}$ is of class $C^{0,1}$. Using Part $5 \mathrm{~b}$ we derive that $\frac{\partial^{2} X}{\partial x \partial t}=\frac{\partial^{2} X}{\partial t \partial x}=\frac{\partial \widehat{\sigma}(t, x)}{\partial t}$. Then we have

$$
\frac{\partial\left(\widehat{\mu}(t, x)-\frac{\partial X}{\partial t}-\frac{1}{2} \frac{\partial \widehat{\sigma}(t, x)}{\partial x}\right)}{\partial x}=\frac{\partial \widehat{\mu}(t, x)}{\partial x}-\frac{\partial \widehat{\sigma}(t, x)}{\partial t}-\frac{1}{2} \frac{\partial^{2} \widehat{\sigma}(t, x)}{\partial x^{2}}=0 .
$$

Proof of the second part of the Main Theorem: Let $Y$ be a recombining process, satisfying for $t \in[0 \cdots T-\delta t]$ the stochastic difference equation

$$
\begin{cases}\delta Y_{t} & =\nu\left(t, Y_{t}\right) \delta t+\sigma\left(t, Y_{t}\right) \delta W_{t} \\ Y_{0} & =x_{0}\end{cases}
$$

where $\nu:\left\{(t, \tilde{Y}(t, x)) \mid(t, x) \in \mathcal{C}_{T}\right\} \rightarrow \mathbb{R}$ is of class $S^{0,1}$ and

$$
\frac{\nu(t, \tilde{Y}(t, x+\delta x))-\nu(t, \tilde{Y}(t, x))}{\delta_{2} \widetilde{Y}(t, x)}
$$

is limited for $(t, x) \in \mathcal{C}_{T-\delta t}$, with $x<t / \sqrt{\delta t}$. Let $X={ }^{\circ} \widetilde{Y}$ and $\mu={ }^{\circ} \nu$. By Theorem 7.1.6 the function $X:[0, T] \times \mathbb{R} \rightarrow \mathbb{R}$ is of class $C^{1,3}$. Also, by Theorem 7.1 .4 the function $\widehat{\mu}:[0, T] \times \mathbb{R} \rightarrow \mathbb{R}$ is of class $C^{0,1}$. By Theorem 7.1.7 we have $\frac{\partial \widehat{\mu}}{\partial x}-\frac{\partial \widehat{\sigma}}{\partial t}-\frac{1}{2} \frac{\partial^{2} \widehat{\sigma}}{\partial x^{2}}=$ 0 . Now

$$
\frac{\partial \widehat{\mu}}{\partial x}=\frac{\partial \mu}{\partial X} \frac{\partial X}{\partial x}=\sigma \frac{\partial \mu}{\partial X}
$$


Also $\frac{\partial \widehat{\sigma}}{\partial x}=\sigma \frac{\partial \sigma}{\partial X}$, hence

$$
\begin{aligned}
\frac{\partial \widehat{\sigma}}{\partial t} & =\frac{\partial \sigma}{\partial t}+\frac{\partial \sigma}{\partial X} \frac{\partial X}{\partial t} \\
& =\frac{\partial \sigma}{\partial t}+\frac{\partial \sigma}{\partial X}\left(\widehat{\mu}-\frac{1}{2} \frac{\partial \widehat{\sigma}}{\partial x}\right) \\
& =\frac{\partial \sigma}{\partial t}+\mu \frac{\partial \sigma}{\partial X}-\frac{\sigma}{2}\left(\frac{\partial \sigma}{\partial X}\right)^{2}
\end{aligned}
$$

and

$$
\frac{\partial^{2} \widehat{\sigma}}{\partial x^{2}}=\frac{\partial}{\partial x}\left(\sigma \frac{\partial \sigma}{\partial X}\right)=\frac{\partial}{\partial X}\left(\sigma \frac{\partial \sigma}{\partial X}\right) \frac{\partial X}{\partial x}=\sigma\left(\frac{\partial \sigma}{\partial X}\right)^{2}+\sigma^{2} \frac{\partial^{2} \sigma}{\partial X^{2}} .
$$

Substituting these equalities in the formula $\frac{\partial \widehat{\mu}}{\partial x}-\frac{\partial \widehat{\sigma}}{\partial t}-\frac{1}{2} \frac{\partial \widehat{\sigma}}{\partial x^{2}}=0$ we obtain (8).

Comment. Theorem 7.1.3d and 7.1.3b are a sort of Ito's Lemma for the recombining process $Y$ : its stochastic variables $Y_{t}$, trend $\nu\left(t, Y_{t}\right)$ and conditional standard deviation $\sigma\left(t, Y_{t}\right)$ are in fact functions of the values taken by the stochastic variables of the Wiener Walk $W$. Indeed,

$$
\begin{aligned}
\nu\left(t, Y_{t}\right) & =\widetilde{\nu}\left(t, W_{t}\right) \simeq \frac{\delta_{1} \tilde{Y}\left(t, W_{t}\right)}{2 \delta t}+\frac{1}{2} \frac{\delta_{2}^{2} \tilde{Y}\left(t, W_{t}\right)}{\delta x^{2}} \\
\sigma\left(t, Y_{t}\right) & =\widetilde{\sigma}\left(t, W_{t}\right) \simeq \frac{\delta_{2} \tilde{Y}\left(t, W_{t}\right)}{\delta x}
\end{aligned}
$$

where $\delta x=x+\delta x-x=\widetilde{W}(t, x+\delta x)-\widetilde{W}(t, x)=\delta_{2} \widetilde{W}(t, x)$.

Conversely, if the trend and the conditional standard deviation of a recombining process $Y$ satisfy (19), by Theorem 7.1 .7 its shadows satisfy $\frac{\partial \widehat{\mu}}{\partial x}-\frac{\partial \widehat{\sigma}}{\partial t}-\frac{1}{2} \frac{\partial^{2} \widehat{\sigma}}{\partial x^{2}}=0$, hence $\sigma \frac{\partial \mu}{\partial X}-\left(\frac{\partial}{\partial t}+\mu \frac{\partial}{\partial X}+\frac{\sigma^{2}}{2} \frac{\partial^{2}}{\partial X^{2}}\right) \sigma=0$ by change of variable.

As a consequence the Main Theorem has the following corollary.

Corollary 7.2 Assume that $T \in \mathbb{T}$ and $x_{0} \in \mathbb{R}$ are standard. Let $\delta t>0, \delta t \simeq 0$ be such that $T / \delta t \in \mathbb{N}$. Let $\sigma:[0, T] \times \mathbb{R} \rightarrow \mathbb{R}$ be standard of class $C^{1,2}$, with $\sigma>0$.

1) Let $\mu:[0, T] \times \mathbb{R} \rightarrow \mathbb{R}$ be standard of class $C^{0,1}$ and let $X_{t}$ satisfy the stochastic difference equation

$$
\left\{\begin{array}{l}
\delta X_{t}=\mu\left(t, X_{t}\right) \delta t+\sigma\left(t, X_{t}\right) \delta W_{t} \\
X_{0}=x_{0}
\end{array}\right.
$$

Assume $\frac{\partial \mu}{\partial X}$ and $\frac{\partial \sigma}{\partial X}$ are bounded and

$$
\sigma \frac{\partial \mu}{\partial X}-\left(\frac{\partial}{\partial t}+\mu \frac{\partial}{\partial X}+\frac{\sigma^{2}}{2} \frac{\partial^{2}}{\partial X^{2}}\right) \sigma=0
$$

Then there exist functions $\tilde{Y}: \mathcal{C}_{T} \rightarrow \mathbb{R}$ of class $S^{1,2}$ and $\widetilde{\nu}: \mathcal{C}_{T} \rightarrow \mathbb{R}$ of class $S^{0,1}$ such that $X$ is nearly equivalent to the process given by the difference equation

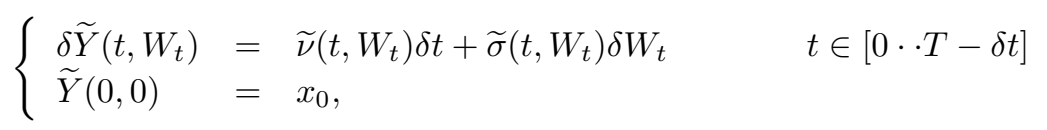


with $\widetilde{\nu}\left(t, W_{t}\right) \simeq \frac{\delta_{1} \widetilde{Y}\left(t, W_{t}\right)}{2 \delta t}+\frac{1}{2} \frac{\delta_{2}^{2} \widetilde{Y}\left(t, W_{t}\right)}{\delta x^{2}}$ and $\widetilde{\sigma}\left(t, W_{t}\right) \simeq \frac{\delta_{2} \widetilde{Y}\left(t, W_{t}\right)}{\delta x}$.

2) Assume there exist functions $\widetilde{Y}: \mathcal{C}_{T} \rightarrow \mathbb{R}$ of class $S^{1,2}$ and $\widetilde{\nu}: \mathcal{C}_{T} \rightarrow \mathbb{R}$ of class $S^{0,1}$ such that $\widetilde{\nu}\left(t, W_{t}\right) \simeq \frac{\delta_{1} \widetilde{Y}\left(t, W_{t}\right)}{2 \delta t}+\frac{1}{2} \frac{\delta_{2}^{2} \widetilde{Y}\left(t, W_{t}\right)}{\delta x^{2}}$ and $\widetilde{\sigma}\left(t, W_{t}\right) \simeq \frac{\delta_{2} \widetilde{Y}\left(t, W_{t}\right)}{\delta x}$. Let $Y$ be the stochastic process defined by the difference equation (20). Put $X={ }^{\circ} \tilde{Y}, \mu={ }^{\circ} \nu$. Then $X:[0, T] \times \mathbb{R} \rightarrow \mathbb{R}$ is of class $C^{1,3}, \mu: X([0, T] \times \mathbb{R}) \rightarrow \mathbb{R}$ is of class $C^{0,1}$, and $\mu$ and $\sigma$ satisfy (8).

\section{References}

[1] S. Albeverio, R. Høegh-Krohn, J.E. Fenstad, T. Lindstrøm, "Nonstandard methods in stochastic analysis and mathematical physics", Pure and Applied Mathematics, Academic Press, Inc., Orlando, Florida vol. 122 (1986).

[2] R.M. Anderson, "A nonstandard representation for Brownian motion and Itô integration,", Israel Journal of Mathematics, vol. 25 (1976) 15-46.

[3] E. Benoît, "Diffusions discrètes et Mécanique Stochastique", Preprint, Centre de Mathématiques Appliquées, École des Mines, Sophia-Antipolis, France vol. (1989) 105p.

[4] E. BEnoît, "Random walks and stochastic differential equations", in: F. and M. Diener (eds.), Nonstandard Analysis in Practice, Springer Universitext vol. (1995) 71-90.

[5] I.P. VAn DEN Berg, "Nonstandard Asymptotic Analysis", Springer Lecture Notes vol. 1249 (1987).

[6] I.P. VAN DEN BERG, "On the relation between elementary partial difference equations and partial differential equations", Ann. of Pure and App. Logic vol. 92 (1998) 235-265.

[7] I.P. VAn DEN BERG, "Principles of infinitesimal Stochastic and Financial Analysis", World Scientific vol. (2000).

[8] I.P. VAN DEN BERG, "Nearly recombining processes and option pricing", in: Perspectives on Econophysics (A. Dionísio, A. Heitor Reis, R. N. Rosa, eds.), Publ. Universidade de Évora, vol. (2007) 35-56.

[9] F. BlaCK, R.M. SCHOLES, "The Pricing of Options and Corporate Liabilities", Journal of Political Economy, vol. 81 (1973) 637-654.

[10] I.P. VAN DEN BERG, F. KoudJeti, "On Binomial Expectations and Option Pricing”, Memorie di Matematica e Applicazioni, vol. 117 XXIII, fasc.1 (1999) 197-212.

[11] J.C. Cox, S. Ross, A. Rubinstein, “ Option Pricing, a simplified approach”, Journal of Financial Economics, vol. 7 (1979) p. 229-263.

[12] N.J. Cutland, "Nonstandard measure theory and its applications", Bull. London Math. Soc., vol. 15 (1983), no. 6, 529-589.

[13] F. Diener, M. Diener, "Nonstandard Analysis in Practice", Universitext, Springer Verlag, vol. (1995).

[14] F. Diener, G. Reeb, “Analyse non standard”, Hermann, Paris, vol. (1985).

[15] H. Gĩ̃o, "Um teorema de DeMoivre-Laplace de ordem arbitrária", Masters Thesis, University of Évora, vol. (2005), 45 p.

[16] H. Doss, "Liens entre équations différentielles stochastiques et ordinaires", Ann. Inst. Henri Poincaré, vol. XIII 2 (1977) p. 99-125.

[17] A.E. Hurd, P. Loeb, "An introduction to nonstandard real analysis", Academic Press, Inc., Orlando, Florida, Pure and Applied Mathematics, vol. 118 (1985). 
[18] H.J. KeISLER, "An infinitesimal approach to stochastic analysis", Mem. Amer. Math. Soc., vol. 48, no. 297 (1984).

[19] F. Koudjeti, I. P. VAN DEN BerG, "Neutrices, external numbers and external calculus", in Nonstandard Analysisin Practice, F. Diener, M.Diener (eds.), Springer universitext, vol. (1995) 145-170.

[20] T. Lindstrøm, “An invitation to nonstandard analysis”, In: Nonstandard analysis and its applications, Cambridge Univ. Press, London Math. Soc. Stud. Texts, vol. 10 (1988) 1-105.

[21] Nelson E., "Internal Set Theory : a new approach to nonstandard analysis", Bull. Amer. Math. Soc., vol. 83 (1977) 1165-1198.

[22] Nelson E., "Radically Elementary Probability Theory", Princeton University Press, (1987).

[23] B. ØKSENDAL, "Stochastic differential equations, an introduction with applications", Springer-Verlag, Berlin, vol. (2003) $6^{\text {th }}$ edition.

[24] PH. E. ProtTER, "Stochastic integration and differential equations", Springer-Verlag, Berlin, Stochastic Modelling and Applied Probability vol. 21 (2005) $2^{\text {nd }}$ edition.

[25] G. REEB, "Internal Équations différentielles et analyse non classique (d'après J.-L.Callot)", Proceedings of the IVth international colloquium of differential geometry, Santiago de Compostella vol. (1978) 240-245.

[26] T. SARI, "Petite histoire de la Stroboscopie", Colloque Trajectorien, Obernai, IRMA Strasbourg (1995) 5-16.

[27] K.D. Stroyan, J.M. BAyod, "Foundations of infinitesimal stochastic analysis", Foundations of infinitesimal stochastic analysis, Studies in Logic and the Foundations of Mathematics vol. 119 (1986). 